\title{
Fate of Quantum Many-Body Scars in the Presence of Disorder
}

\author{
Ian Mondragon-Shem $\odot,{ }^{1,}{ }^{*}$ Maxim G. Vavilov $\odot,{ }^{2}$ and Ivar Martin $\circledast^{1}$ \\ ${ }^{1}$ Materials Science Division, Argonne National Laboratory, Argonne, Illinois 08540, USA \\ ${ }^{2}$ Department of Physics, University of Wisconsin-Madison, Madison, Wisconsin 53706, USA
}

(Received 2 November 2020; accepted 20 July 2021; published 23 September 2021)

\begin{abstract}
Experiments performed on strongly interacting Rydberg atoms have revealed surprising persistent oscillations of local observables. These oscillations have been attributed to a special set of nonergodic states, referred to as quantum many-body scars. Although these states have enriched our understanding of thermalization in quantum systems, their interplay with disorder in realistic quantum simulators has remained unclear. We address this question by studying numerically and analytically the magnetization and spatiotemporal correlators of a scar model of disordered and interacting qubits that can be realized in present-day simulators. While the oscillation amplitudes of these observables decay with time as the disorder strength is increased, their oscillation frequency remains remarkably constant. This stability stems from resonances of the disordered spectrum in the overlap with the clean scar eigenstates that are approximately centered at the same scar energies of the clean system. We also find that multiple additional sets of scar resonances become accessible due to the presence of disorder and further enhance the oscillation amplitudes. Our results show the robustness of nonergodic dynamics in scar systems, and opens the door to understanding their behavior in near-term quantum devices and potentially using them as a resource in quantum-sensing protocols to calibrate quantum hardware.
\end{abstract}

DOI: 10.1103/PRXQuantum.2.030349

\section{INTRODUCTION}

Recent progress in the design and coherent control of programmable quantum simulators has made it possible to discover new and striking nonequilibrium phenomena [1-4]. A particularly remarkable example has been the detection of quantum many-body scars using a 51-qubit cold-atom platform [4]. In this system, strongly interacting Rydberg atoms were initially prepared in a product state and subsequently allowed to evolve under their own unitary dynamics. Unexpectedly, the dynamics of measured observables presented persistent oscillations at finite energy density. This represented a violation of the eigenstate thermalization hypothesis (ETH) [5], a central tenet of statistical mechanics. The ETH says that states at finite energy density evolve at long times into ergodic states wherein the expectation value of local observables converge to thermal values. The experimental observation of persistent oscillations thus signals that nonergodic

\footnotetext{
*ian.mondragonshem@northwestern.edu
}

Published by the American Physical Society under the terms of the Creative Commons Attribution 4.0 International license. Further distribution of this work must maintain attribution to the author(s) and the published article's title, journal citation, and DOI. behavior is at play and that physics beyond the ETH was accessed by the Rydberg quantum simulator.

Subsequent analyses revealed that this phenomenon shared similarities with the previously known concept of quantum scarring [6,7]. This concept was originally conceived to describe single-particle wave functions that are anomalously concentrated around unstable periodic orbits of classically chaotic systems. A consequence of this are periodic revivals in the wave-function fidelity for initial Gaussian wave packets $[8,9]$. In the many-body case realized in the Rydberg simulator, it was similarly found that the initial product state remained concentrated in a restricted part of the Hilbert space spanned by a small set of energy eigenstates embedded in an ergodic spectrum (which had been studied previously in a different context in Ref. [10]). These special eigenstates are nearly equally spaced in energy (with separation $\omega_{\text {scar }}$ ) and approximately span the initial product state used experimentally. The observed persistent oscillations at the frequency $\omega_{\text {scar }}$ thus follow from these properties, which motivated referring to these special eigenstates as quantum many-body scars.

These states have garnered intense interest and have been found in a wide range of systems, both static and driven, as well as in higher dimensions [11-21]. Several insights have been obtained by constructing exact scar eigenstates and from analytical results [22-29]. Strategies have also been devised to understand and enhance 
emergent dynamical symmetries in scar subspaces, which are sufficient to explain the persistent oscillations of observables in interacting systems [30-34]. Furthermore, fundamental questions about the nature of such scar states have been addressed by studying their proximity to integrability, as well as connections with concepts of quantum chaos [35-38].

The existence of quantum many-body scars could have far-reaching consequences for fundamental and practical reasons. Generic mechanisms that inhibit thermalization in quantum systems at finite energy density are few and far in between. Each time a new mechanism is discovered, we gain further insights into the nature of nonequilibrium quantum many-body systems. Furthermore, such nonergodic phenomena could be leveraged to control chaotic behavior in the processing of quantum information, specially as quantum devices continue to be scaled up.

In this regard, an important question that has not been addressed yet is the interplay between many-body scarring and the disorder that is present in realistic quantum simulators. Disorder has been a central theme across the main quantum simulators. Superconducting circuits, for example, are naturally affected by unwanted uncontrollable qubit-to-qubit variations [39-41], and in certain cases artificial "disorder" is introduced on purpose to avoid frequency collisions when implementing cross-resonance gates [42]. Trapped ions and Rydberg atoms have also been leveraged to study disordered systems such as many-body localized phases and time crystals [43-45]. To fully understand the role quantum scars could play in the development of quantum technologies, it is thus essential that we understand the impact that spatial imperfections can have on their nonergodic properties.

The possibility, in particular, of bringing together two highly nontrivial mechanisms that avoid thermalization - quantum scars and many-body localization (MBL) - is tantalizing. Many-body localization was first predicted for sufficiently strong disorder in a general class of interacting electronic systems [46]. Further studies of nonequilibrium phenomena in the presence of disorder have shown that MBL and its associated phases of matter persist in a wide variety of interacting systems even far from equilibrium [44,47-55]. In the space of models, systems with scars reside somewhere between the integrable and fully ergodic systems. It is therefore natural to expect their response to disorder to yield a spectrum of unusual features, both in and out of equilibrium. This is indeed what we find in this work.

While disorder in scarred systems was discussed in Refs. [56-58], it was only in the context of fine-tuned models where disorder was designed not to affect the scar states themselves. The effects of disorder in the PXP model were studied in Ref. [59], but the focus was solely on many-body localization physics and not on the nonergodic dynamics arising from quantum scars. Thus, the broader question of the effects of generic disorder on quantum scars has remained open. One might expect that the randomizing effect of a disordered environment will generically suppress the oscillation amplitude and distort the oscillation frequency of observables. On the other hand, one could also expect that at least some quantum scars will be robust against disorder given that strong kinematic constraints underlie their existence in some models. These constraints introduce strong spatiotemporal correlations, suggesting enhanced robustness with respect to spatial imperfections.

In this work we use a prototypical model of interacting qubits that can be realized in Rydberg atoms, trapped ions, and superconducting circuits. The model system is schematically represented in Fig. 1(a). As will be clear from our discussion, our results can be extended to other scarred models as well. As a first step, we find that eigenstate diagnostics of scar states [6] fail when disorder is turned on. However, clear oscillations can still be identified in observables such as the magnetization of the system as well as spatiotemporal correlators. While disorder indeed leads to the decay of these oscillations with time, their frequency remains close to $\omega_{\text {scar }}$ for a finite range of disorder strengths, exhibiting significant rigidity to generic perturbations.

We provide a physical picture for these observations by deriving analytically the disorder-averaged dynamics of the magnetization and temporal correlators in the weak-disorder limit. This derivation shows that, instead of remaining discrete eigenstates, scar states become transformed into resonances in the energy spectrum of the disordered system. These are resonances of the disordered spectrum in the overlap with the clean scar eigenstates. Given the clear connection to the scar eigenstates of the clean limit, we dub them scar resonances, with the understanding that they are not themselves eigenstates of the disordered system and are thus not scar states in the traditional sense [8,60-62]. For sufficiently small disorder strength, these resonances have widths smaller than $\omega_{\text {scar }}$, and are centered at the energies of the clean scars states. As a result, the observables we probe continue to oscillate at the scar frequency before decaying irreversibly. Surprisingly, we also uncover multiple additional scar resonances not associated with the previously known scar states; these novel states come into play in the presence of disorder, and, remarkably, enhance the magnetization oscillations at intermediate times. While in the bulk of this work we focus on the regime of strongly interacting qubits (the so called "PXP" regime), at the end we fully map out the dynamical regimes of the model for arbitrary coupling strength as a function of disorder.

Our results show that quantum many-body scars continue to have an impact in moderately disordered quantum simulators. The robustness of the oscillation frequency and the multiple sets of scar resonances we have uncovered serve as a new window into the underlying complexities of 
scarred systems that could help further understand the circumstances under which they arise. These properties can also potentially prove to be valuable, for example, in the calibration of quantum hardware. We indeed discuss an example application of quantum scars as a resource for quantum metrology.

\section{Outline}

We now outline the structure of this work and summarize the main results. We begin in Sec. II by introducing the model we use as a case study; the system is shown schematically in Fig. 1(a). We discuss basic aspects of this model, including how scars arise in the strongly interacting limit, and also the type of disorder we consider in this work.

In Sec. III, we consider the effects of disorder on the eigenstate properties of the PXP model. We discuss how scar states are no longer detectable in the energy spectrum (Fig. 2) and determine how the ergodicity of the system is

(a)
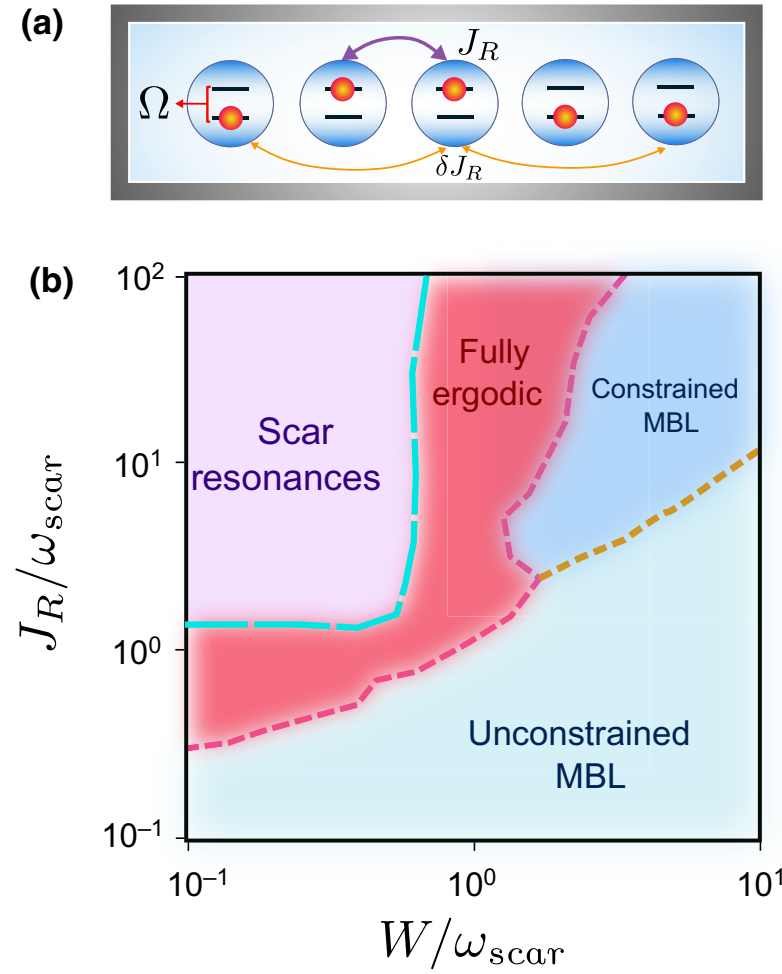

FIG. 1. (a) Schematic representation of the simulator modeled by Eq. (1). The excited and ground states of each qubit are separated by an energy $\Omega$. Only when two neighboring qubits are in their excited states will the qubits interact, with energy $J_{R}$. When $J_{R} \gg \Omega$, a kinematic constraint develops that leads to the formation of quantum many-body scars. (b) Diagram of the dynamical regimes obtained in this work as a function of the nearest-neighbor interaction $J_{R}$ and disorder strengths $W$ : a regime that exhibits scar dynamics due to scar resonances, a regime that is ergodic without scar resonances, a constrained MBL phase, and a MBL without kinematic constraints. affected (Fig. 3). We then move on in Sec. IV to carefully analyze the magnetization of the system. We show that it continues to carry signatures of nonergodic oscillations at the scar frequency for moderate disorder strengths due to the kinematic constraint of the PXP model (Figs. 5-7).

In order to understand these results, in Sec. V we derive explicitly the disorder-averaged magnetization dynamics in the weak disorder limit. This derivation shows that the dynamics is controlled by disorder-induced resonances that arise from multiple towers of scar states of the clean system. Although their widths grow with disorder strength and this leads to the decay of the oscillation amplitude, nonergodic oscillations continue to manifest in the magnetization as long as the width remains smaller than the scar frequency (Fig. 9). We further show in Sec. VI that scar resonances also account for temporal correlations in the system (Figs. 11 and 12).

We bring everything together in Sec. VII by showing a diagram in the parameter space of interaction and disorder strengths, which shows four distinct dynamical regimes [Fig. 1(a)]: a regime with scar resonances, a regime that is ergodic and does not exhibit revival dynamics, a constrained many-body localized phase, and another many-body localized phase without kinematic constraints. Finally, in Sec. VIII we discuss how our results can be tested in various quantum simulators and also be used for quantum-sensing applications, and in Sec. IX we discuss the main conclusions from this work and propose future directions of research.

\section{MODEL}

In this section, we introduce the model that will serve as a case study to understand how quantum many-body scars respond to disorder. We begin with the Hamiltonian that models the original simulator in which scars are detected

$$
H_{R}=\sum_{r=1}^{L}\left[\frac{\Omega}{2} \sigma_{r}^{x}+J_{R} P_{r}^{+} P_{r+1}^{+}\right],
$$

where the Pauli matrices $\sigma_{r}^{x, y, z}$ act on ground and excited qubit states $\{|\bullet\rangle,|0\rangle\}$, respectively, we define the projection operator $P_{r}^{ \pm}=\frac{1}{2}\left(\mathbb{I} \pm \sigma_{r}^{z}\right)$, and we assume periodic boundary conditions. This Hamiltonian models a chain of qubits, each driven resonantly with strength $\Omega$ (Rabi frequency) and interacting with strength $J_{R}$ when two nearest neighbors are in the excited state $[63,64]$. While this Hamiltonian is inspired by Rydberg simulators $[6,7,14,65]$, it can be programmed in other simulators such as superconducting (SC) circuits and trapped ions as we discuss later.

In the strongly interacting limit $J_{R} \gg \Omega$, there is a large energy cost $J_{R}$ to go from the state $|\cdots \circ \bullet \circ \circ \cdots\rangle$ to the state $|\cdots \circ \bullet \bullet \circ \cdots\rangle$, which has two neighboring qubits in their excited state. As a result, when $J_{R} \gg \Omega$, the energy 
spectrum splits into $L$ bands. The Hilbert space spanned by each of these bands is characterized by a fixed number $n_{\text {exc }}$ of pairs of contiguous qubits in their excited state. When the system is initialized within one of these bands, the number $n_{\text {exc }}$ is thus preserved by the dynamics.

In particular, the product state $\left|\mathbb{Z}_{2}\right\rangle=|\circ \bullet \ldots \circ \bullet\rangle$, which we use as the initial condition in this work, satisfies $n_{\text {exc }}=0$. The effective Hamiltonian that generates the dynamics within this subspace, referred to as the PXP model [6], is given by

$$
H_{\mathrm{PXP}}=\frac{\Omega}{2} \sum_{r=1}^{L} \mathcal{P} \sigma_{r}^{x} \mathcal{P},
$$

where $\mathcal{P}=\prod_{r}\left(\mathbb{I}-P_{r}^{+} P_{r+1}^{+}\right)$projects into the $n_{\mathrm{exc}}=0$ sector. The resulting Hamiltonian differs from that of a paramagnet by the presence of projection operators. To understand the impact on the dynamics of these projectors, we can express the Hamiltonian in the equivalent form $H_{\text {PXP }}=(\Omega / 2) \sum_{r=1}^{L} P_{r-1}^{-} \sigma_{r}^{x} P_{r+1}^{-}$, which holds if we consider the dynamics of initial states that satisfy $\mathcal{P}|\Psi(0)\rangle=$ $|\Psi(0)\rangle$. This form of the Hamiltonian illustrates that, while in a paramagnetic system the operator $\sigma_{r}^{x}$ generates rotations of the qubit at the position $r$ without regard to the state of other qubits, in the strongly interacting case this rotation can happen only when the qubits located at the positions $(r-1)$ and $(r+1)$ are in their respective ground states.

One striking consequence of this kinematic constraint is the large (although incomplete) and periodic revival dynamics of product states such as the $\left|\mathbb{Z}_{2}\right\rangle$ state. Initially, it was thought that strong interactions could provide sufficiently generic relaxation mechanisms such that the eigenstate thermalization hypothesis would hold. In particular, the ETH dictates that an initial state at finite energy density should evolve under Eq. (1) into a thermal state at long times. However, the persistent and periodic revival of the $\mathbb{Z}_{2}$ state directly challenged these assumptions. Spectrally, this can be understood in terms of a set of $L+1$ special eigenstates of the PXP model, referred to as quantum scars, which are embedded in a presumed ergodic spectrum within the $n_{\text {exc }}=0$ subspace. They are approximately equally spaced by an energy

$$
\omega_{\text {scar }}=2 \pi v_{\text {scar }}=\eta \Omega, \quad \eta \approx 0.636 .
$$

Scar states have an exceptionally large overlap with the $\mathbb{Z}_{2}$ state, and exhibit less-than volume-law spatial entanglement even though they reside at finite energy density. The properties of these special eigenstates explain the nonergodic revival dynamics of the $\left|\mathbb{Z}_{2}\right\rangle$ state.

Now, the return probability of the $\mathbb{Z}_{2}$ state obtained by evolving with the PXP model is only partial. Furthermore, its spatial entanglement entropy grows with time, which suggests that the quantum scars of the conventional PXP model exhibit some level of hybridization with ergodic states. To correct for this, a suitable deformation $\delta H$ can be added to the Hamiltonian that removes this growth in entanglement entropy and further optimizes the return probability of an initial $\mathbb{Z}_{2}$ to reach unity [30]. One can think of this ideal limit of optimized scars as a reference point for understanding the impact of disorder on its nonergodic dynamics. We use the particular deformation

$$
\delta H=\sum_{r=1}^{L} \delta J_{R}\left(\sigma_{r+2}^{z}+\sigma_{r-2}^{z}\right) \mathcal{P} \sigma_{r}^{x} \mathcal{P},
$$

where $\delta J_{R}=-0.026 \Omega$ [30]. Longer-ranged terms further improve the quality of scar states, but for our present purposes this deformation is sufficient. While we refer to an ideal limit of perfect scar eigenstates for conceptual clarity, our main conclusions also follow from the conventional PXP Hamiltonian. As a result, a simulator need not realize this deformation, although it could certainly be engineered, for example, in digital simulators.

The main subject of our work is the effect of disorder on the scar states, the corresponding revivals, and order parameter oscillations. The disorder that we introduce is generic in that it breaks all the spin and translational symmetries of the clean PXP model; namely, we consider the operator

$$
\hat{W}=\sum_{r, a} h_{a}(r) \sigma_{r}^{a} .
$$

The random fields $h_{a}(r)$ with $a=x, y, z$ are chosen uniformly distributed in the range [-W/2, $W / 2]$, where $W$ measures the strength of the disorder. The presence of all Pauli matrices breaks the symmetries that the clean PXP model has, such as chiral and time-reversal symmetries.

Throughout most of this work we mainly study the strongly interacting limit. This effectively projects the disorder operator into the $n_{\mathrm{exc}}=0$ Hilbert space. As a result, the full Hamiltonian is given by

$$
H=H_{\mathrm{PXP}}+\delta H+\mathcal{P} \hat{W} \mathcal{P} \text {. }
$$

Later, in Sec. VII, we lift this strong constraint to study the behavior of the system as a function of interaction strength $J_{R}$ in the full Hilbert space.

When disorder is introduced into the system, the fields in Eq. (5) will tend to make the qubits in the system precess around random directions and at different rates. As a result, there will be a competition between the desynchronizing effect introduced by the disorder potential and the clean correlated oscillatory dynamics of the PXP Hamiltonian. Understanding the outcome of this competition is the main goal of this work. 


\section{Spectral properties}

In this section, we study the impact of disorder on spectral and eingenstate properties of the Hamiltonian (6) that are commonly attributed to the existence of nonergodic dynamics of scar systems. We present evidence that scars can no longer be clearly identified as eigenstates of the system for moderate disorder strengths. Furthermore, we discuss how disorder initially enhances the ergodicity of the system at low disorder, and eventually induces a many-body localization transition at strong disorder.

\section{A. Destruction of scar eigenstates}

A basic question that first arises when disorder is introduced is whether scars can still be identified as eigenstates in the spectrum of the system. One standard way to identify scar states is to single out those eigenstates that have a high overlap with the $\mathbb{Z}_{2}$ state [6,7]. In Fig. 2(a), we show the distribution of this overlap for $W=0$ and $W=0.25 \omega_{\text {scar }}$ using a fixed disorder realization. While in the clean limit it is easy to identify states that have large overlap with the $\mathbb{Z}_{2}$ state, it is difficult to do so when disorder is turned on. Disorder has hybridized the clean-limit scar states with other spectrally near-by states to such an extent that the $\mathbb{Z}_{2}$ state no longer appears to be concentrated in a small subset of states. As a result, there are no clear signs of scar eigenstates in this overlap diagnostic.

Further evidence that scar eigenstates are absent in the spectrum can be obtained by calculating the spatial entanglement entropy of each energy eigenstate. If we divide the system in two halves $A$ and $B$, the entanglement entropy
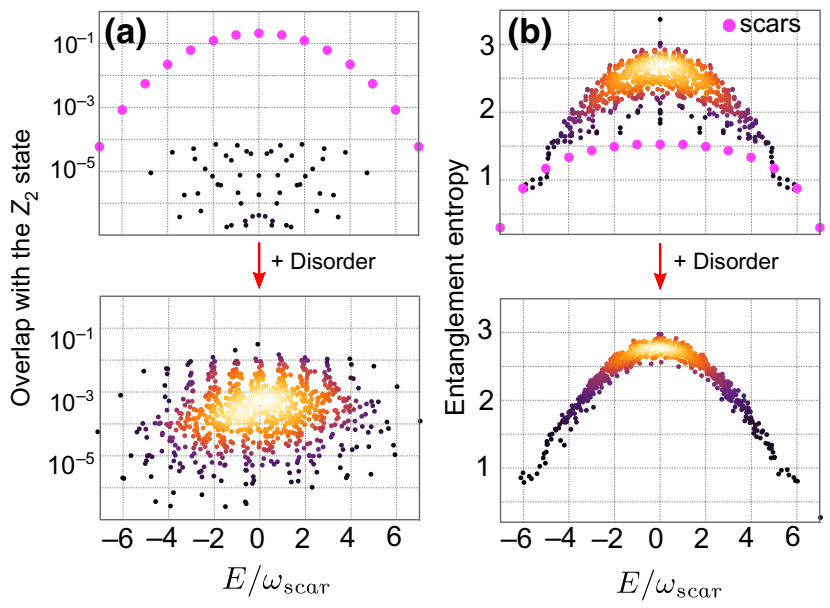

FIG. 2. (a) Overlap with the $\mathbb{Z}_{2}$ state, and (b) entanglement entropy for each eigenstate with $L=14$. Top figures correspond to the clean system, where clear signatures of scar states appear at the integer values of $E / \omega_{\text {scar }}$. The bottom figures correspond to disorder strength $W=0.25 \omega_{\text {scar }}$ for a particular disorder realization. The scar signatures have become difficult to identify after disorder is introduced in the system. of a state $\left|E_{n}\right\rangle$ is $\mathcal{S}\left(E_{n}\right)=-\operatorname{Tr}\left\{\rho_{A}^{(n)} \ln \rho_{A}^{(n)}\right\}$, where $\rho_{A}^{(n)}=$ $\operatorname{Tr}_{B}\left\{\left|E_{n}\right\rangle\left\langle E_{n}\right|\right\}$ and $\operatorname{Tr}_{B}$ traces the degrees of freedom in region $B$. When the entanglement entropy is shown for each eigenstate in the system in the clean limit, as seen in Fig. 2(b), it is possible to clearly discern isolated scar eigenstates that appear to have less-than volume-law entanglement. Upon introducing disorder, however, the states of low entanglement recede quickly into the full set of volume-law ergodic states. Thus, we again see that hybridization of optimized scars with ergodic states has erased signatures of scar states in the entanglement of energy eigenstates.

\section{B. Enhanced ergodicity and localization transition}

In addition to obscuring signatures of scar eigenstates as we have seen in the previous section, disorder can also lead to other kinds of nonergodic behavior such as many-body localization [48,51]. It is thus pertinent that we examine how the ergodicity of the system changes as the disorder strength is varied. To this end, we calculate the mean value of the level spacing ratios [47]

$$
r_{n}=\frac{\min \left(\delta E_{n+1}, \delta E_{n}\right)}{\max \left(\delta E_{n+1}, \delta E_{n}\right)},
$$

where $\delta E_{n}=E_{n+1}-E_{n}$ with the energy eigenvalues $\left\{E_{n}\right\}$ sorted in ascending order. Since the disorder operator we have chosen does not respect any symmetries, the disordered Hamiltonian we are studying belongs to the Gaussian unitary ensemble (GUE), implying that $\left[\left\langle r_{n}\right\rangle\right] \approx 0.6$ when the system is ergodic [66], where $\langle\cdot\rangle$ denotes spectral averaging. When the system is localized, such as in a MBL phase, Poisson statistics dictates that $\left[\left\langle r_{n}\right\rangle\right] \approx 0.38$.

We present $\left[\left\langle r_{n}\right\rangle\right]$ in Fig. 3(a) as a function of system size. The flow with system size reveals signatures of a localization transition at around $W_{\text {Th-L }} \approx 2.13 \omega_{\text {scar }}$. When $W<W_{\text {Th-L }}$, the approach to the transition point does not occur monotonically for finite sizes. Instead, the system presents an enhanced approach to the ergodic value $\left[\left\langle r_{n}\right\rangle\right]=0.6$ at a disorder strength somewhat below $W_{\text {Th-L }}$. When $W>W_{\text {Th-L }}$, notice that $\left[\left\langle r_{n}\right\rangle\right]$ converges quite slowly to the Poisson value at strong disorder, which is the value that is expected for a many-body localized phase. This is clearly due to the kinematic constraint imposed by the presence of projection operators. Slow convergence to the Poisson value was also obtained in Ref. [59] for a similar disordered PXP model, where this type of manybody localization under kinematic constraints was dubbed constrained MBL.

Since level statistics at low disorder can be affected by the symmetries of the clean system, we also compute an additional diagnostic of ergodicity that makes use of the eigenstates of the system to confirm these results. The ETH states that the expectation value of local observables $\hat{\mathcal{O}}$ 
(a)

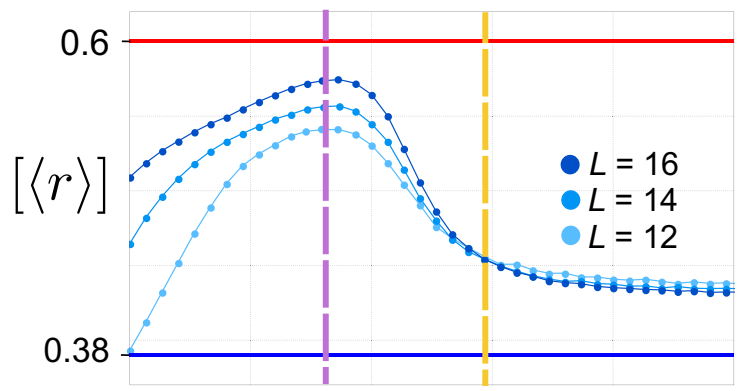

(b)

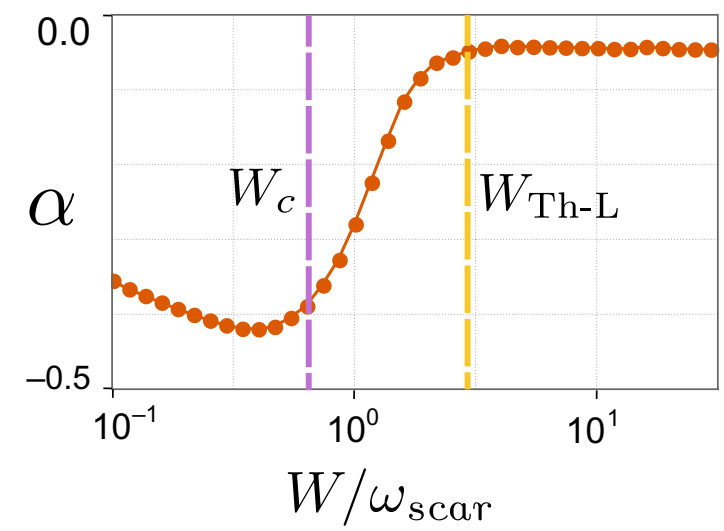

FIG. 3. (a) Mean level spacing ratios $\left[\left\langle r_{n}\right\rangle\right]$ defined by Eq. (7) and (b) decay exponent of $\Delta \hat{\mathcal{O}}$ defined by Eq. (8) for the domainwall operator $\hat{\mathcal{O}}=\sigma_{L / 2}^{z} \sigma_{L / 2+1}^{z}$, as a function of disorder strength. The error bars in both figures are of the order or less than the circle markers. The disorder strength $W_{c}$ at which nonergodic oscillations are lost is denoted by the purple line, whereas the disorder strength $W_{\text {Th-L }}$ at which the constrained MBL phase sets in is denoted by the yellow line. "Th-L" refers to the thermal-to-localized transition.

varies smoothly with energy. As a result, contiguous states satisfy

$$
\Delta \mathcal{O}=\left|\left\langle E_{n}|\hat{\mathcal{O}}| E_{n}\right\rangle-\left\langle E_{n-1}|\hat{\mathcal{O}}| E_{n-1}\right\rangle\right| \sim e^{-\mathcal{S}\left(E_{n}\right) / 2},
$$

where $\mathcal{S}(E)$ is the entropy at energy $E$. A particular choice one can consider is the domain-wall operator $\hat{\mathcal{O}}=$ $\sigma_{L / 2}^{z} \sigma_{L / 2+1}^{z}$. This operator was used to study signatures of integrability in the clean PXP model [36]. Near infinite temperature, we have $\mathcal{S}(E) \sim \ln \mathcal{D}$, where $\mathcal{D}$ is the dimension of the Hilbert space. This implies that $\Delta \hat{\mathcal{O}} \sim \mathcal{D}^{-1 / 2}$ if the system is ergodic. We proceed as in Ref. [36], and we calculate $\Delta \hat{\mathcal{O}} \sim \mathcal{D}^{\alpha}$ as a function of $\mathcal{D}$ to extract the exponent $\alpha$. For ergodic systems, the exponent $\alpha$ should thus converge to $-1 / 2$, whereas in the nonergodic regime we expect $\Delta \hat{\mathcal{O}}$ to decay slower with $|\alpha|<1 / 2$. We show the exponent $\alpha$ extracted by using $L=12,14,16$ in Fig. 3(b). The exponent approaches $-1 / 2$ below $W_{\text {Th-L }}$, and is significantly suppressed above it, consistent with our findings using energy-level statistics.

\section{SYSTEM DYNAMICS}

The results from the previous section raise the question of whether nonergodic dynamics can still be detected in the presence of disorder. To explore this question, we turn to the study of the magnetization of the system. We define the on-site magnetization as

$$
\mathcal{M}_{a}(r, t)=\left\langle\Psi(t)\left|\sigma_{r}^{a}\right| \Psi(t)\right\rangle
$$

where $|\Psi(t)\rangle$ is the time-evolved state obtained from solving the Schrodinger equation with the initial condition $|\Psi(0)\rangle=\left|\mathbb{Z}_{2}\right\rangle$. We further define the Fourier transforms $\widetilde{\mathcal{M}}_{a}(k, t)=\left\langle\Psi(t)\left|\widetilde{\sigma}_{k}^{a}\right| \Psi(t)\right\rangle, \overline{\mathcal{M}}_{a}(k, \omega)=\int_{0}^{\infty} d t e^{-i \omega t}$ $\widetilde{\mathcal{M}}_{a}(k, t)$, where

$$
\widetilde{\sigma}_{k}^{a}=\sum_{r=1}^{L} e^{i k r} \sigma_{r}^{a} .
$$

In what follows, we will first calculate the magnetization in the clean limit in order to understand how it depends on the presence of scar eigenstates. We then investigate its behavior when disorder is introduced.

\section{A. Scar signatures in the clean limit}

Let us begin by writing the initial state as $|\Psi(0)\rangle=$ $\sum_{l} \psi_{l}\left|\phi_{l}^{(0)}\right\rangle$, where $\left\{\left|\phi_{l}^{(0)}\right\rangle\right\}$ is the set of eigenstates of the clean system. Since the time-evolved state is given by $|\Psi(t)\rangle=\sum_{l} e^{-i E_{l}^{(0)} t} \psi_{l}\left|\phi_{l}^{(0)}\right\rangle$, the magnetization takes the form

$$
\widetilde{\mathcal{M}}_{a}(k, t)=\sum_{l, l^{\prime}} e^{i \omega_{l^{\prime} l^{t}}} \psi_{l^{\prime}}^{*} \psi_{l}\left\langle\phi_{l^{\prime}}^{(0)}\left|\widetilde{\sigma}_{k}^{a}\right| \phi_{l}^{(0)}\right\rangle
$$

where we define $\omega_{l^{\prime} l}=E_{l^{\prime}}^{(0)}-E_{l}^{(0)}$. The calculation of the magnetization hinges on understanding the behavior of the matrix elements $\left\langle\phi_{l^{\prime}}^{(0)}\left|\widetilde{\sigma}_{k}^{a}\right| \phi_{l}^{(0)}\right\rangle$.

Although the behavior of these matrix elements is not known for arbitrary wave vector $k$, progress can be made for the particular case when $k=\pi$ and the initial state is $|\Psi(0)\rangle=\left|\mathbb{Z}_{2}\right\rangle[30,65]$. Given that the $\mathbb{Z}_{2}$ state is approximately spanned by optimized scar states, we need only to consider matrix elements $\left\langle\phi_{l^{\prime} \in \Lambda_{s}}^{(0)}\left|\sigma_{\pi}^{a}\right| \phi_{l \in \Lambda_{s}}^{(0)}\right\rangle$, where $\Lambda_{s}$ is the set of indices of optimized scar states. In particular, approximate ladder operators $\sigma_{s}^{ \pm}$can be defined within the scar subspace where

$$
\sigma_{s}^{ \pm}=\frac{1}{2}\left(\widetilde{\sigma}_{\pi}^{z} \mp i \eta^{-1} \widetilde{\sigma}_{\pi}^{y}\right),
$$

which connect scar states that differ by an energy $\pm \omega_{\text {scar }}$ (see Appendix A for details). The existence of these emergent ladder operators are sufficient for the occurrence of persistent oscillations at a fixed frequency in the dynamics 
of integrable and nonintegrable systems [33,34]. Indeed, the matrix elements $\left\langle\phi_{l^{\prime} \in \Lambda_{s}}^{(0)}\left|\sigma_{s}^{ \pm}\right| \phi_{l \in \Lambda_{s}}^{(0)}\right\rangle$ that contribute to the magnetization are those for which $\omega_{l^{\prime} l}= \pm \omega_{\text {scar }}$. Plugging this into Eq. (11) with $k=\pi$ leads to the magnetization components

$$
\begin{aligned}
\widetilde{\mathcal{M}}_{y}(\pi, t) & \approx \eta L \sin \left(\omega_{\text {scar }} t\right), \\
\widetilde{\mathcal{M}}_{z}(\pi, t) & \approx-L \cos \left(\omega_{\text {scar }} t\right),
\end{aligned}
$$

which exhibit oscillations at the scar frequency with fixed amplitude. These magnetization oscillations capture the nonergodic dynamics induced by the presence of scars in the spectrum, and could thus be useful observables to track any remaining signatures of scar physics when the system becomes disordered.

Now, when disorder is introduced, other states of the clean system outside of the scar subspace will inevitably become populated. As a result, in the following sections we need to also understand the behavior of matrix elements $\left\langle\phi_{l^{\prime} \notin \Lambda_{s}}^{(0)}\left|\sigma_{s}^{ \pm}\right| \phi_{l \notin \Lambda_{s}}^{(0)}\right\rangle$. Not much has been said in the literature, however, about their behavior. These states have been largely assumed to be ergodic, which might suggest that they do not contribute to oscillations at the scar frequency. Consider, however, Fig. 4(a), where we show a density plot of all the matrix elements $\left|\left\langle\phi_{l^{\prime}}^{(0)}\left|\widetilde{\sigma}_{\pi}^{y}\right| \phi_{l}^{(0)}\right\rangle\right|$, with $l, l^{\prime}$ ordered according to the energy of the corresponding state (we included the set of optimized scars). The structure of this matrix reveals that, while most entries are vanishingly small, there are some that are exceptionally large. At first glance there is no apparent order to the distribution of these large matrix elements. However, a clear pattern emerges when one performs an appropriate reordering of the energy basis by grouping them into sets that are connected via the same ladder operators $\sigma_{s}^{ \pm}$. This reordered basis reveals that the energy eigenstates can be organized into towers labeled by an index $J$, with each tower containing a number $\mathcal{D}_{J}$ of states labeled by an index $m$. There are a few states near the middle of the spectrum that do not seem to form towers with any other state, so they form sets with a single state each; such sets do not contribute appreciably to the magnetization.

Using this reordering, we again show $\left|\left\langle\phi_{J^{\prime} m^{\prime}}^{(0)}\left|\widetilde{\sigma}_{\pi}^{y}\right| \phi_{J m}^{(0)}\right\rangle\right|$ in Fig. 4(b) (details of how we performed this reordering are presented in Appendix B). The matrix is approximately block diagonal with respect to $J$, and each block is tridiagonal, so we approximately write

$$
\left\langle\phi_{J^{\prime} m^{\prime}}^{(0)}\left|\widetilde{\sigma}_{\pi}^{a}\right| \phi_{J m}^{(0)}\right\rangle \approx \delta_{J^{\prime} J}\left(\Gamma_{J m}^{a,+1} \delta_{m^{\prime} m \pm 1}+\Gamma_{J m}^{a,-1} \delta_{m^{\prime} m-1}\right),
$$

for $a=y, z$. In this expression, we define $\Gamma_{J m}^{a, \kappa}=$ $\left\langle\phi_{J m+\kappa}^{(0)}\left|\widetilde{\sigma}_{\pi}^{a}\right| \phi_{J m}^{(0)}\right\rangle$. Similar to the case of optimized scars (which in our notation corresponds to the $J=1$ tower), this tridiagonal structure selects frequencies in Eq. (11) that are close to $\omega_{\text {scar }}$ for most towers. As we see in the following section, upon introducing disorder, these additional towers will become populated and their nonergodic oscillations near the scar frequency will be revealed.

We note that it is remarkable that the PXP model is comprised of multiple towers of scar states, of which the set of optimized scar states is just one example. One of the reasons that such towers have remained hidden is, in part, because the $\mathbb{Z}_{2}$ state is spanned largely by only the $J=1$ tower. The multitower structure of the PXP model can lead to nonergodic $\mathrm{SU}(2)$ dynamics with a variety of other product states, e.g., $\mathbb{Z}_{3}$ state, which is spanned by a different set of scar towers and requires suitably defined observables, as we discuss elsewhere [67].

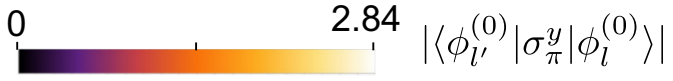

(a)

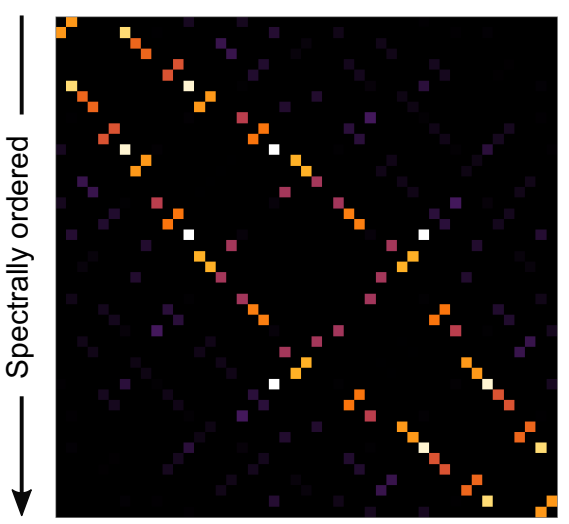

(b)

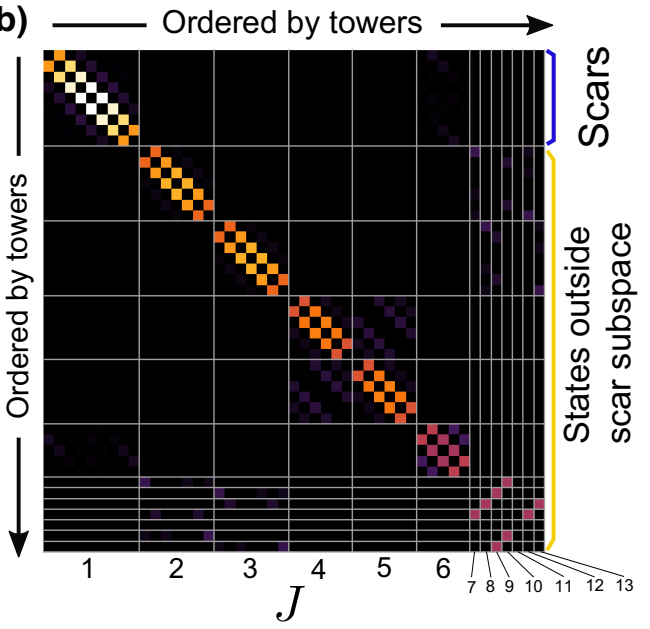

FIG. 4. Density plot of the matrix elements $\left|\left\langle\phi_{l^{\prime}}^{(0)}\left|\widetilde{\sigma}_{\pi}^{y}\right| \phi_{l}^{(0)}\right\rangle\right|$ with respect to the basis of energy eigenstates ordered by (a) energy and (b) towers, respectively, for $L=8$. The corresponding figure for $\left|\left\langle\phi_{l^{\prime}}^{(0)}\left|\widetilde{\sigma}_{\pi}^{z}\right| \phi_{l}^{(0)}\right\rangle\right|$ is similar. 


\section{B. Stability of oscillations at the scar frequency}

Let us now examine the stability of magnetization oscillations at the scar frequency. We begin by calculating the on-site magnetization $\left[\mathcal{M}_{z}(r, t)\right]$ and its Fourier distribution $\left[\left|\overline{\mathcal{M}}_{z}(k, \omega)\right|\right]$ for a few values of the disorder strength, as shown in Fig. 5. Here, and throughout this work, all disorder-averaged quantities shown are averaged over 500 disorder realizations and are denoted by square brackets $[\cdot]$. As can be seen in Fig. 5, for the weak disorder case $W=0.1 \omega_{\text {scar }}$, there is a clear periodic pattern in both space and time. Temporally, each qubit oscillates as a function of time at the scar frequency, initially between the maximum amplitudes $\left[\mathcal{M}_{z}(r, t)\right]= \pm 1$ and gradually decaying with time. Spatially, the even and odd sites appear antiferromagnetically ordered throughout the dynamics, as expected from the kinematic constraint of the PXP model. This leads to sharp peaks in $\left[\left|\overline{\mathcal{M}}_{z}(k, \omega)\right|\right]$ at $(k, \omega)=\left(\pi, \pm \omega_{\text {scar }}\right)$ as shown in the right column of Fig. 5, consistent with Eq. (13). As the disorder strength is increased, the magnetization amplitude decays faster in time, until there is no longer any clear oscillation at the scar frequency for sufficiently strong disorder. Correspondingly, the Fourier peaks broaden, especially in the frequency domain. It is clear, however, from both $\left[\mathcal{M}_{z}(r, t)\right]$ and $\left[\left|\overline{\mathcal{M}}_{z}(k, \omega)\right|\right]$, that the frequency of oscillation continues to be dominated by $\omega=\omega_{\text {scar }}$ for a finite range of disorder strengths even though the qubits are experiencing random and biased fields. This indicates that the dynamics of the system continues to exhibit signatures of many-body scars. For strong enough disorder, the distribution eventually focuses at $(k, \omega)=(\pi, 0)$, indicating that the dynamics of the system has been rendered temporally trivial while maintaining antiferromagnetic ordering.

In order to better understand how stable the oscillations remain at the scar frequency, it is useful to compare $\left[\left|\overline{\mathcal{M}}_{z}(\pi, \omega)\right|\right]$ for both the deformed PXP and the paramagnetic case $J_{R}=\delta J_{R}=0$. The paramagnetic limit is equivalent to removing the projection operators from the PXP model. Because of this, the contrast between the two systems can shed light on the possible stabilizing effects that the kinematic constraint has on the oscillations of the scar system. In Figs. 6(a) and 6(b) we show $\left[\left|\overline{\mathcal{M}}_{z}(\pi, \omega)\right|\right]$ for both limits as a function of disorder strength. A sharp difference is revealed in their response to the presence of disorder. In the paramagnetic case, the dominant frequency of oscillation at the lowest disorder strength $W=0.1 \omega_{\text {scar }}$ we show in this figure is centered at $\omega=\Omega$, although it is already visibly broadened at this disorder strength. As $W$ is increased, the broadening grows continuously, to the point that there is no clear dominant frequency of oscillation. As expected, the ease with which the dynamics develops a broad range of frequencies is the manifestation of each qubit oscillating at its own local disorder-induced Zeeman field.

By contrast, in the strongly interacting case there is a clear persistent dominant frequency of oscillation centered around $\omega=\omega_{\text {scar }}$. This occurs in spite of the qubits experiencing the same random fields as those used in the paramagnetic case. The strong kinematic constraint that correlates the rotation of a given qubit with respect to its

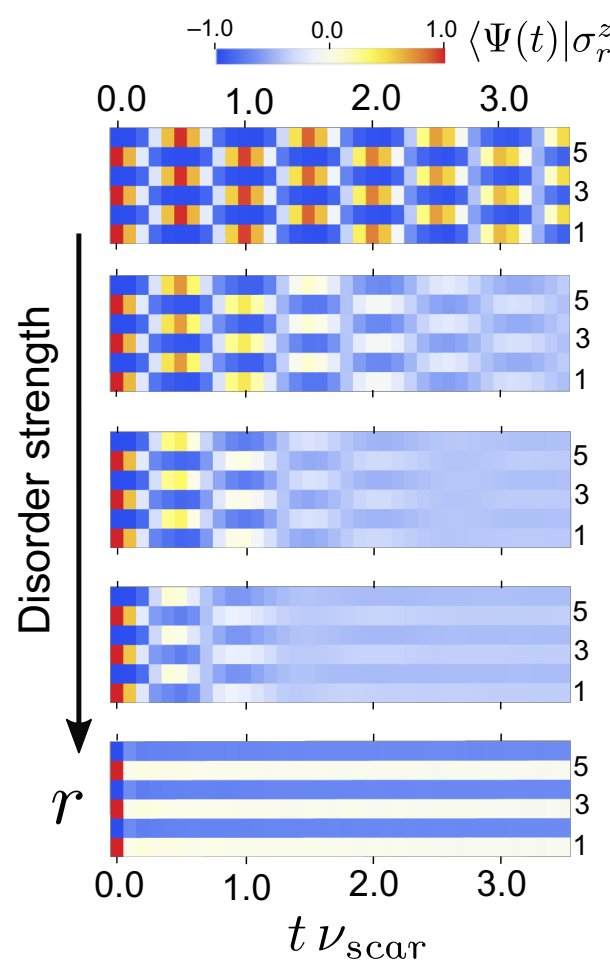

$\sigma_{r}^{z}|\Psi(t)\rangle$

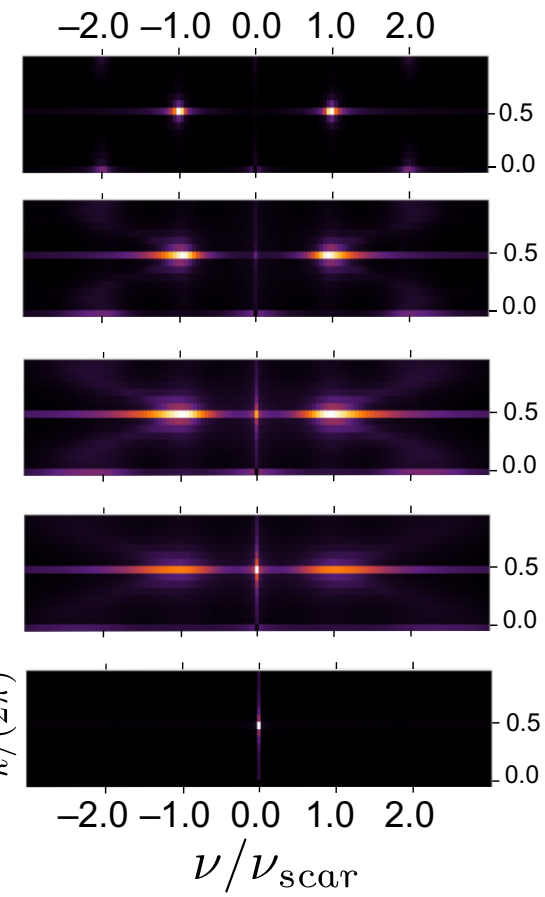

FIG. 5. Left: time evolution of $\left[\mathcal{M}_{z}(r, t)\right]$ for disorder strengths $W / \omega_{\text {scar }}$ $=0.1,0.19,0.63,1.58,7.41$. In these figures, $L=16$, but we show only the first six sites for visual clarity. Right: corresponding disorder-averaged Fourier component $\left[\left|\overline{\mathcal{M}}_{z}(k, \omega)\right|\right]$ at the same disorder strengths. For clarity, we subtract the component $\left[\left|\overline{\mathcal{M}}_{z}(0,0)\right|\right]$, which represents a constant homogeneous shift in the on-site magnetization. For weak disorder, oscillations occur at $(k, \omega)=\left(\pi, \omega_{\text {scar }}\right)$. As the disorder strength is increased, the distribution broadens, and eventually concentrates at $(k, \omega)=(\pi, 0)$. 


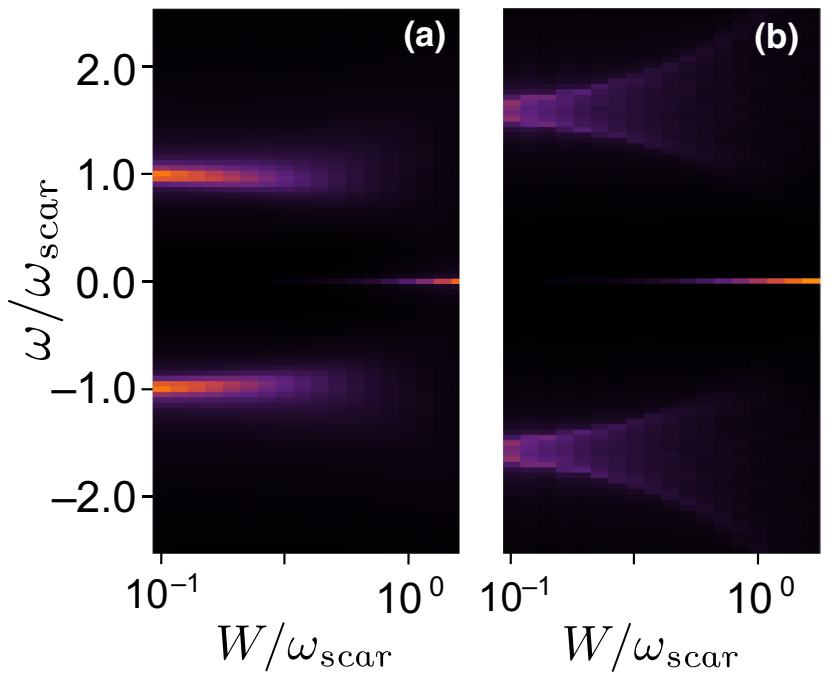

FIG. 6. Fourier component $\left[\left|\overline{\mathcal{M}}_{z}(\pi, \omega)\right|\right]$ as a function of $\omega$ and $W$ for the PXP model (a) and the free paramagnet (b). A pronounced broadening occurs in the paramagnetic case, whereas in the PXP model the dynamics is consistently centered and localized at the scar frequency.

neighbors continues to robustly enforce oscillations at the scar frequency. As a check of the stability of this observation, we find that the oscillations at the scar frequency persist when the system size is increased, as we exemplify in Fig. $7(\mathrm{a})$, where $\left[\left|\overline{\mathcal{M}}_{z}(\pi, \omega)\right|\right] / L$ does not change appreciably for $L=12,14,16$ with $W=0.1 \omega_{\text {scar }}$. We thus find clear evidence that, even though the magnetization amplitude decreases with time, the scarred system continues to sustain oscillations at the scar frequency for a significant range of disorder strengths - a highly nontrivial effect induced by the strong interactions between qubits. Such behavior occurs even though conventional eigenstate diagnostics clearly fail.

Oscillations at the scar frequency terminate at a characteristic disorder strength $W_{c}$. This can be seen in Fig. 6(a), where there appears to be a marked change in dynamical regimes wherein a strong signal in $\left[\left|\widetilde{\mathcal{M}}_{z}(\pi, \omega)\right|\right]$ eventually yields to a clear peak at zero frequency when the disorder strength is increased. To better visualize this, in Fig. $7\left(\right.$ b) we show $\left[\left|\widetilde{\mathcal{M}}_{z}\left(\pi, \omega_{\text {scar }}\right)\right|\right]$ and $\left[\left|\widetilde{\mathcal{M}}_{z}(\pi, 0)\right|\right]$ as a function of disorder strength. At weak but nonzero disorder, the dynamics is dominated by $\left[\left|\widetilde{\mathcal{M}}_{z}\left(\pi, \omega_{\text {scar }}\right)\right|\right]$. As the disorder strength is increased, the peak at $\omega_{\text {scar }}$ gradually decreases, while at the same time the zero-frequency component increases, eventually rendering the time evolution trivial at strong disorder. The change between both regimes can be taken to occur when both frequency components have the same magnitude, namely around $W_{c} \approx 0.63 \omega_{\text {scar }}$. As can be seen in this figure, the crossing between both curves does not appear to change as the system size is increased.
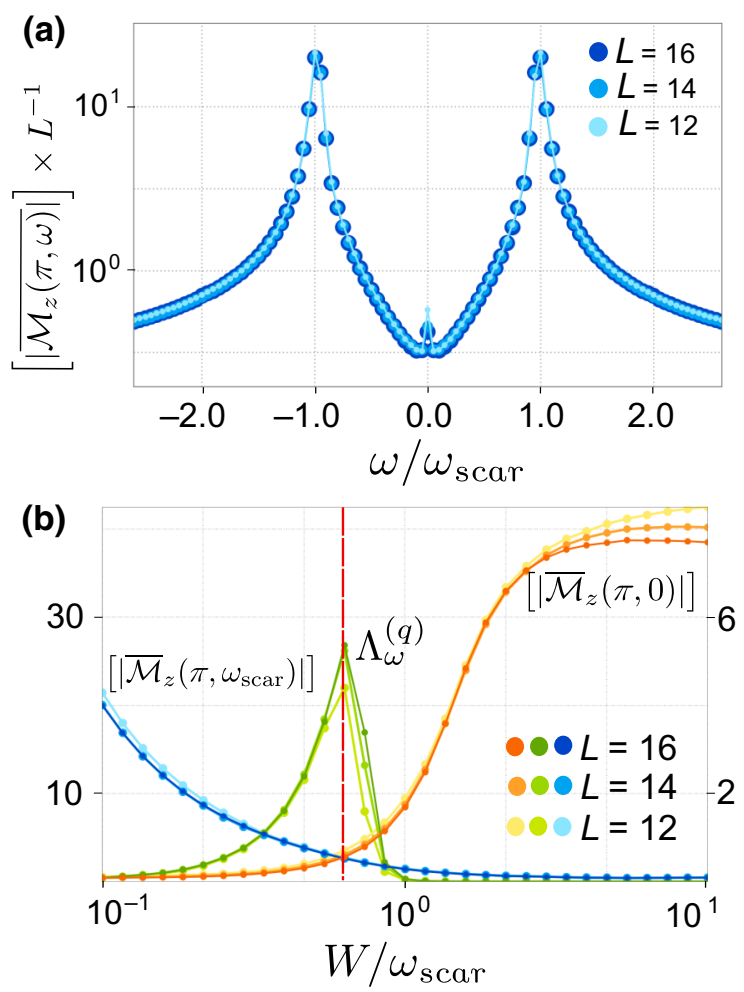

FIG. 7. (a) Behavior of $\left|\overline{\mathcal{M}}_{z}(\pi, \omega)\right| / L$ as the system size is increased with $W=0.1 \omega_{\text {scar }}$, showing that there are no appreciable changes. (b) Behavior as a function of disorder strength (for three system sizes) of the magnetization Fourier components $\left[\left|\overline{\mathcal{M}}_{z}\left(\pi, \omega_{\text {scar }}\right)\right|\right]$ and $\left[\left|\overline{\mathcal{M}}_{z}(\pi, 0)\right|\right]$ (values indicated on the left axis); and the normalized frequency distribution $\Lambda_{\omega}^{(q=6)}$ (values indicated on the right axis). There is a clear and stable transition between a regime with scar dynamics and a dynamically trivial regime. In this figure, we choose $q=6$ for convenience as it accentuates the position of the peak, but other values of $q$ also work.

To capture this transition more clearly, we make use of the normalized frequency distribution $[55,68] \mathcal{F}(\omega)=$ $\frac{1}{\mathcal{N}}\left[\left|\widetilde{\mathcal{M}}_{z}(\pi, \omega)\right|\right]$, where $\mathcal{N}=\sqrt{\int d \omega\left[\left|\widetilde{\mathcal{M}}_{z}(\pi, \omega)\right|\right]^{2}}$. We introduce the quantity $\Lambda_{\omega}^{(q)}=\left[\int d \omega \mathcal{F}^{q}(\omega)\right]^{-1}$, which measures the spread of $\mathcal{F}(\omega)$ in the frequency domain. This is analogous to the participation ratio defined in real space to study the spatial localization of wave functions.

As we show in Fig. 7(b), $\Lambda_{\omega}^{(q=6)}$ is suppressed at both weak and strong disorder, as expected since the system is strongly dominated by $\omega=\omega_{\text {scar }}$ and $\omega=0$, respectively. At intermediate disorder strengths, $\Lambda_{\omega}^{(q)}$ develops a peak at $W_{c}$ that does not appear to shift significantly with system size. This peak is present for other values of $q \neq 6$ as well, so our particular choice for $q$ is somewhat arbitrary. It is used in this work because it yields a sharper peak than those obtained with lower values of $q$ (higher values can make it even sharper). The peak signals a spread of 
the frequency components at $W_{c}$, allowing us to identify two distinct dynamical regimes, one with clear oscillations at the scar frequency and another that is manifestly featureless.

These results thus show that oscillations at the scar frequency persist over an appreciable time scale even as disorder is making the system more ergodic. It is clear that the increased ergodicity induced by disorder is consistent with the temporal decay of the magnetization amplitude. What is nontrivial is that these oscillations remain close to $\omega_{\text {scar }}$, indicating temporal rigidity.

Given that the characteristic disorder strengths $W_{c}$ and $W_{\text {Th-L }}$ appear to be distinct (as indicated in Fig. 3), there are three distinct dynamical regimes that arise as a function of disorder strength: a regime with oscillations at the scar frequency, a fully thermal phase, and a constrained MBL phase. In the following section, we present a quantitative interpretation for these observations that will show that oscillations at the scar frequency occur due to the presence of scar resonances in the spectrum.

\section{SCAR RESONANCES}

The behavior of the magnetization poses a number of questions regarding the presence of scars in the disordered system. Scars no longer exist as eigenstates in the spectrum, and yet the magnetization shows multiple oscillations in an increasingly ergodic system before decaying at long times. Central to the underlying dynamics is the fact that disorder couples optimized scars with the rest of the spectrum. This brings into focus, on the one hand, the manner in which scars hybridize with nearby ergodic states and, on the other, the role that states outside of the scar subspace play in the decay of the magnetization signal.

As we see in this section, the robustness in the oscillations occurs in fact via two mechanisms. First, we present evidence that scars become transformed into resonances with a nonzero width that depends on the level of disorder in the simulator. As time goes by, the finite life time of these resonances leads to their decay into other states in the spectrum. Additionally, although the states outside the scar subspace are more highly entangled and ergodic, they also lead to oscillations close to the same scar frequency, contributing appreciably to the magnetization of the system.

To begin our description of the problem, suppose we initialize the system in the $\mathbb{Z}_{2}$ state, which can be accurately expressed as $|\Psi(0)\rangle=\sum_{l \in \Lambda_{s}} \psi_{l}\left|\phi_{l}^{(0)}\right\rangle$, where $\Lambda_{s}$ is the set of indices of optimized scar states. We express the time-evolved state in the basis of clean eigenstates in the form $|\Psi(t)\rangle=\sum_{l} \mathcal{A}_{l}(t) e^{-i E_{l}^{(0)} t}\left|\phi_{l}^{(0)}\right\rangle$, where the sum runs over the full set of energy eigenstates of the clean system since disorder will inevitably induce transitions to states outside of the scar subspace. To satisfy the initial conditions, we must have that $\mathcal{A}_{l \in \Lambda_{s}}(0)=\psi_{l}$, and $\mathcal{A}_{l \notin \Lambda_{s}}(0)=0$.
The disorder-averaged magnetization is then

$$
\left[\widetilde{\mathcal{M}}_{a}(\pi, t)\right]=\sum_{l l^{\prime}} e^{i \omega_{l^{\prime}} t}\left[\mathcal{A}_{l^{\prime}}^{*}(t) \mathcal{A}_{l}(t)\right]\left\langle\phi_{l^{\prime}}^{(0)}\left|\widetilde{\sigma}_{\pi}^{a}\right| \phi_{l}^{(0)}\right\rangle
$$

The magnetization is thus determined by the dynamics of $\left[\mathcal{A}_{l^{\prime}}^{*}(t) \mathcal{A}_{l}(t)\right]$ as well as the structure of the matrix elements $\left\langle\phi_{l^{\prime}}^{(0)}\left|\widetilde{\sigma}_{\pi}^{a}\right| \phi_{l}^{(0)}\right\rangle$. Any deviations from the clean limit will arise from the factor $\left[\mathcal{A}_{l^{\prime}}^{*}(t) \mathcal{A}_{l}(t)\right]$ in Eq. (14). This factor can change the oscillation amplitude as well as the oscillation frequency of the magnetization $\left[\widetilde{\mathcal{M}}_{a}(\pi, t)\right]$.

\section{A. Decay of optimized scar amplitudes}

Insight into the behavior of the amplitudes $\mathcal{A}_{l \in \Lambda_{s}}(t)$ can be gained by calculating their dynamics in the weakdisorder limit $W \ll \omega_{\text {scar }}$. When the disorder strength is weak, scar eigenstates primarily hybridize with nearby states within a spectral neighborhood of size $\Delta \ll \omega_{\text {scar }}$, as we schematically represent in Fig. 8(a). Since the number of states in this spectral neighborhood is exponentially large in the system size, the coupling of the scar state to its spectral surrounding leads to the irreversible decay of the amplitude $\mathcal{A}_{l \in \Lambda_{s}}(t)$. The dynamics corresponds to the decay of a discrete state that is embedded in a quasicontinuum. Standard perturbative calculations of this type of decay [69] lead us to derive the approximate expression for the amplitude (see Appendix $\mathrm{C}$ for details):

$$
\mathcal{A}_{l \in \Lambda_{s}}(t) \approx \psi_{l} e^{-i \delta \mathcal{E}_{l}(t) t} e^{-(1 / 2) \lambda_{l}(t) t},
$$

where we define

$$
\begin{aligned}
\delta \mathcal{E}_{l}(t)= & \left\langle\phi_{l}^{(0)}|\hat{W}| \phi_{l}^{(0)}\right\rangle \\
& +\sum_{\substack{l^{\prime} \notin \Lambda_{s} \\
\left|\omega_{l l^{\prime}}\right|<\Delta}} \frac{\left|\left\langle\phi_{l}^{(0)}|\hat{W}| \phi_{l^{\prime}}^{(0)}\right\rangle\right|^{2}}{\omega_{l l^{\prime}}}\left(1-\frac{\sin \omega_{l l^{\prime}} t}{\omega_{l l^{\prime}} t}\right), \\
\lambda_{l}(t)= & 2 \sum_{\substack{l^{\prime} \notin \Lambda_{s} \\
\left|\omega_{l l^{\prime}}\right|<\Delta}}\left|\left\langle\phi_{l}^{(0)}|\hat{W}| \phi_{l l^{\prime}}^{(0)}\right\rangle\right|^{2}\left(\frac{1-\cos \omega_{l l^{\prime}} t}{\omega_{l l^{\prime}}^{2} t}\right) .
\end{aligned}
$$

The expression Eq. (16) represents the dynamics induced by a general disorder operator $\hat{W}$, although below we use the explicit form defined in Eq. (5). The factor $e^{-i \delta \mathcal{E}_{l}(t) t}$ has fixed magnitude and encodes perturbative shifts in the scar energy due to the disorder potential. By contrast, the factor $e^{-(1 / 2) \lambda_{l}(t) t}$ leads to the decay of the scar amplitude. In fact, at sufficiently long times, the factor $\left(1-\cos \omega_{l l^{\prime}} t\right) /\left(\omega_{l l^{\prime}}^{2} t\right)$ is highly peaked at low frequencies and converges to $\sim \pi \delta\left(\omega_{l l^{\prime}}\right)$. The sum over states in Eq. (16b) can then be turned into an integral that effectively yields the decay rate of the scar state as determined 


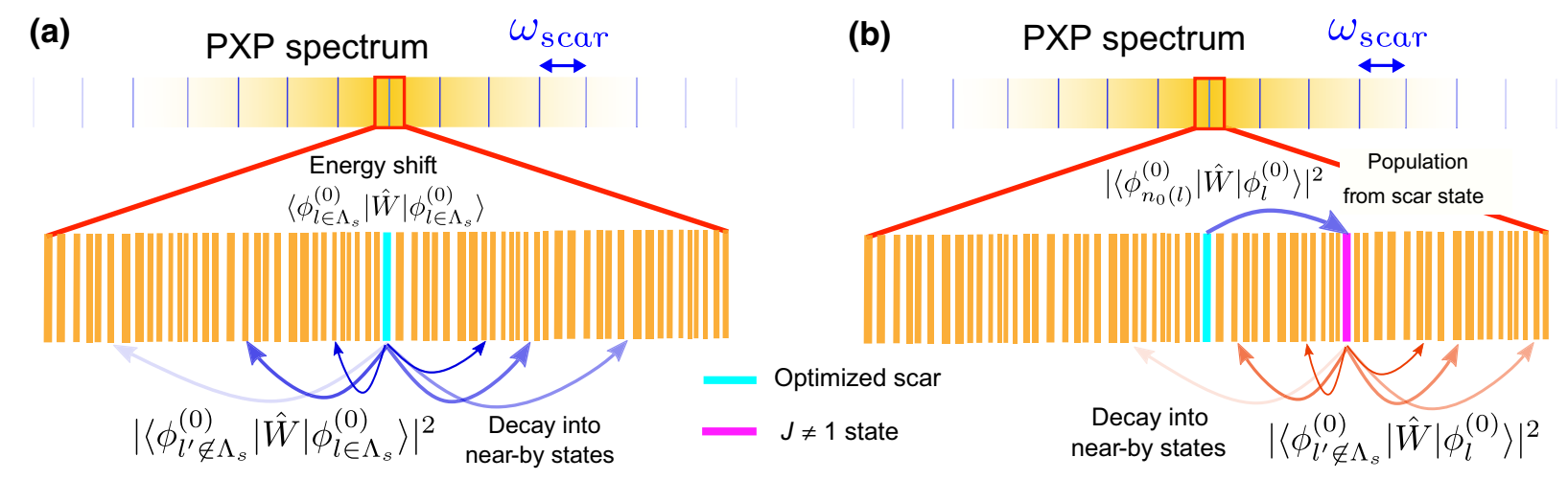

FIG. 8. (a) Direct decay of scar states. The disorder induces transitions from the scar state into spectrally nearby generic states, with the rate controlled by matrix elements of the form $\left|\left\langle\phi_{l^{\prime} \notin \Lambda_{s}}^{(0)}|\hat{W}| \phi_{l \in \Lambda_{s}}^{(0)}\right\rangle\right|^{2}$. Additionally, there is an energy shift of the form $\left\langle\phi_{l \in \Lambda_{s}}^{(0)}|\hat{W}| \phi_{l \in \Lambda_{s}}^{(0)}\right\rangle$. (b) Decay via $J \neq 1$ tower states. The initial scar state couples (blue arrow) to a state in a separate tower (purple line), which then decays (blue arrows) into the spectrally nearby generic states. These processes give significant contribution [comparable to the main channel in (a)] to the order parameter dynamics at finite times.

by Fermi's golden rule. We do not take this limit here since we are dealing with finite-sized systems with discrete spectra and, as we see, the expressions in terms of discrete sums account very well for the observed dynamics.

The next step is to calculate the average $\left[\mathcal{A}_{l^{\prime} \in \Lambda_{s}}^{*}(t)\right.$ $\left.\mathcal{A}_{l \in \Lambda_{s}}(t)\right]$ over realizations of the random fields $h_{a}(r)$ in Eq. (5). To perform this average explicitly, we make use of a Gaussian probability distribution with the same first and second moments, $\left[h_{a}(r)\right]=0$ and $\left[h_{a}^{2}(r)\right]=W^{2} / 12$ respectively, of the box disorder distribution we use numerically. We are thus led to the explicit disorder-averaged expression

$$
\begin{aligned}
{\left[\mathcal{A}_{l^{\prime} \in \Lambda_{s}}^{*}(t) \mathcal{A}_{l \in \Lambda_{s}}(t)\right] \approx } & \psi_{l^{\prime}}^{*} \psi_{l} \sqrt{\operatorname{det}\left\{\alpha_{l^{\prime} l}(t)\right\}} \\
& \times e^{-\left(W^{2} t^{2} / 24\right)\left(\rho_{l^{\prime} l^{\prime}}-\rho_{l l}\right) \cdot \alpha_{l^{\prime} l^{\prime}}(t) \cdot\left(\rho_{l^{\prime} l^{\prime}}-\rho_{l l}\right)},
\end{aligned}
$$

where we define $\left(\rho_{l l^{\prime}}\right)_{n}=\left\langle\phi_{l}^{(0)}\left|\sigma_{r(n)}^{a(n)}\right| \phi_{l^{\prime}}^{(0)}\right\rangle$, with $n=$ $1, \ldots, 3 L, a(n)=(n-1) \bmod 3, r(n)=\left\lfloor\frac{n-1}{3}\right\rfloor$, we made the identification $\sigma_{r}^{1,2,3}=\sigma_{r}^{x, y, z}$, and

$$
\begin{aligned}
\alpha_{l l^{\prime} l}^{-1}(t) & =\mathbb{I}+\frac{W^{2}}{6}\left\{G_{l^{\prime}}^{\dagger}(t, 0)+G_{l}(t, 0)\right\}, \\
G_{l}\left(t_{1}, t_{2}\right) & =\sum_{\substack{l^{\prime} \neq l, l^{\prime} \notin \Lambda_{s} \\
\left|\omega_{l l^{\prime}}\right|<\Delta}}\left(\rho_{l l^{\prime}}^{*} \cdot \rho_{l l^{\prime}}^{T}\right) f_{m n}\left(t_{1}, t_{2}\right),
\end{aligned}
$$

where $f_{m n}\left(t, t^{\prime}\right)=i \omega_{m n}^{-2}\left\{\omega_{m n}\left(t-t^{\prime}\right)-i\left[\exp \left(i \omega_{\mathrm{mn}} t^{\prime}\right)-\exp \right.\right.$ $\left.\left.\left(i \omega_{m n} t\right)\right]\right\}$. We define this function in terms of two temporal arguments for convenience, as it arises again in this form in the following section. For the same reason, although the sum in Eq. (19) has the redundant constraints $l^{\prime} \neq l$ and $l^{\prime} \notin \Lambda_{s}$, it will be useful later when we discuss the amplitudes $\mathcal{A}_{l \notin \Lambda_{s}}(t)$.
The expression Eq. (17) shows us that the magnetization decays on account of both the square root and exponential factors, encoding two sources of decay. The exponential factor represents dephasing induced by random energy shifts from Eq. (16a), which are linear in the disorder strength. The factor $\sqrt{\operatorname{det}\left\{\alpha_{l^{\prime} l}(t)\right\}}$ arises in part from the exponential factor in Eq. (15), which encodes information about the finite life time of the scar state as it decays into its spectral neighborhood.

On the other hand, the magnetization oscillation frequency can also change if $\left[\mathcal{A}_{l^{\prime} \in \Lambda_{s}}^{*}(t) \mathcal{A}_{l \in \Lambda_{s}}(t)\right]$ develops a time-dependent imaginary component. Inspection of Eq. (17) reveals that this can happen via the second-order energy shift in Eq. (16a). Since this shift is suppressed with respect to the first-order term in the energy shift, we expect deviations from $\omega_{\text {scar }}$ to be small. This explains why, for the disorder strengths in Fig. 6(a), which are small with respect to $\omega_{\text {scar }}$, there appears to be some level of rigidity in the frequency of oscillation of the magnetization.

We now use Eq. (17) to compute the contribution of the scar states to the magnetization using the restricted sum

$$
\left.\left[\widetilde{\mathcal{M}}_{a}(\pi, t)\right]\right|_{\Lambda_{s}}=\sum_{m=1}^{L} 2 \operatorname{Re}\left(e^{i \Omega_{1 m} t} \mathcal{Q}_{1 m}(t) \Gamma_{1 m}^{a,+1}\right),
$$

where we define $\mathcal{Q}_{J m}(t)=\left[\mathcal{A}_{J m+1}^{*}(t) \mathcal{A}_{J m}(t)\right]$, and $\Omega_{J m}=$ $\omega_{J m+1, J m}$. In Fig. 9(a) we present $\left.\left[\widetilde{\mathcal{M}}_{a}(\pi, t)\right]\right|_{\Lambda_{s}}$ for the numerically obtained values and our explicit disorderaveraged result. In calculating the analytic result, the matrix elements $\rho_{l l^{\prime}}$ are calculated numerically using the eigenstates of the clean system. Both match very closely, which confirms that our basic picture of the decay of scar amplitudes is accurate. When compared, however, with 


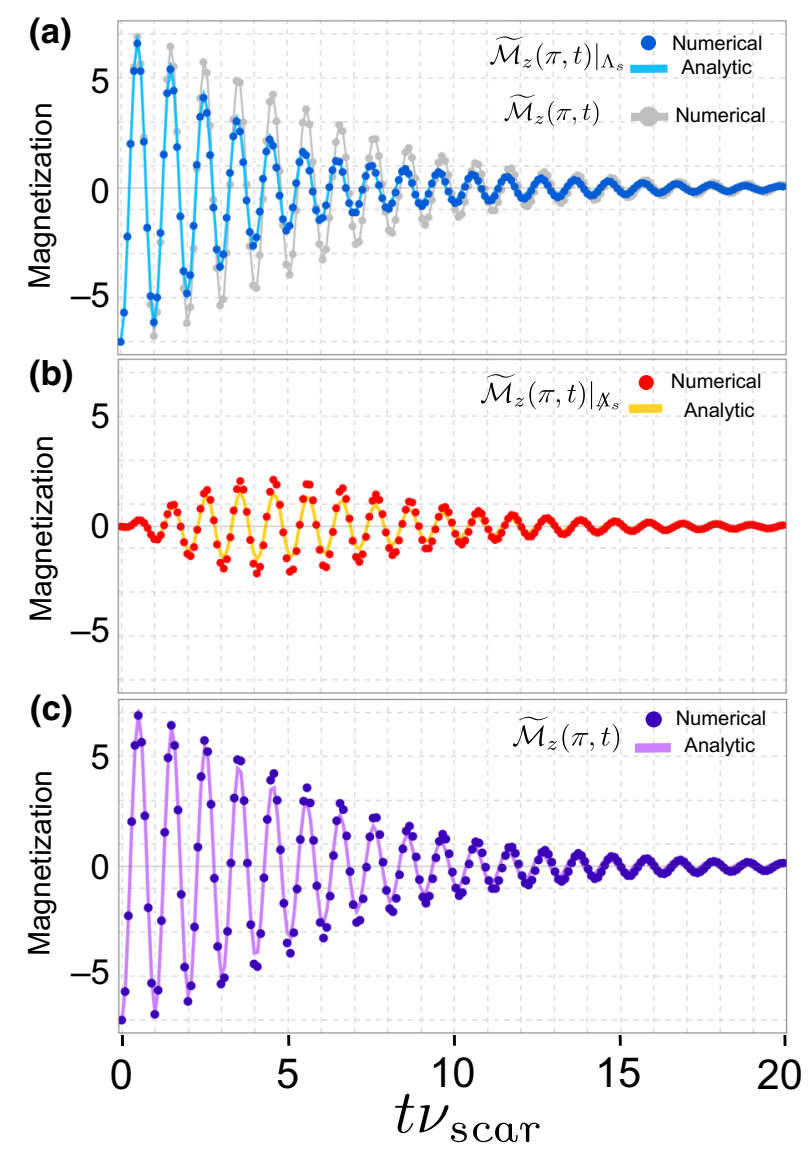

FIG. 9. Comparison of contributions to the magnetization $\left[\widetilde{\mathcal{M}}_{z}(\pi, t)\right]$ when evaluated exactly numerically (dots) and analytically (solid lines) for $L=14$ and $W=0.1 \omega_{\text {scar. The matrix }}$ elements $\rho_{l l^{\prime}}$ are calculated numerically using the eigenstates of the clean system. (a) Contribution from optimized scar states, $J=1$ tower, with the analytic curve obtained using Eq. (21). (b) Contribution from states outside the main scar subspace, decay via $J \neq 1$ towers as shown in Fig. 8, with the corresponding analytic curve obtained from Eq. (24). (c) Contribution from all states in the spectrum, with the corresponding analytic curve. In (a), we also show the net magnetization by gray dots (the connecting gray line is a guide for the eye) to illustrate that the contribution from the optimized scar states is not sufficient to account for the observed dynamics.

the full magnetization signal, this contribution is not sufficient to account for the full magnetization of the system, even though it clearly is sufficient in the clean limit. The inevitable conclusion is that states outside of the scar subspace are contributing to the dynamics of the system. What is perhaps most remarkable is that this additional contribution must oscillate at the same frequency $\omega_{\text {scar }}$, even though it involves states that are known to be more ergodic and more highly entangled than the optimized scar states. This requires us to investigate how such states can coherently contribute to the observed magnetization, as we do so in the following section.

\section{B. Decay of $J \neq 1$ scar amplitudes}

The key to identifying the missing contribution to the magnetization can be gleaned from Eq. (14). It is clear that as the scar states become depopulated, amplitudes for states outside the scar subspace will inevitably begin to grow. In order to obtain the dynamics arising from the other towers, the decay of the amplitudes $\mathcal{A}_{l \notin \Lambda_{s}}(t)$ can be calculated similar to the case of optimized scars, as we schematically represent in Fig. 8(b). Following the same main steps we took for the set of optimized scars, the solution for $\mathcal{A}_{l \notin \Lambda_{s}}(t)$ can be written in the integral form (see Appendix $\mathrm{C}$ for details)

$$
\begin{aligned}
\mathcal{A}_{l \notin \Lambda_{s}}(t) \approx & -i\left\langle\phi_{l}^{(0)}|\hat{W}| \phi_{n_{0}(l)}^{(0)}\right\rangle \int_{0}^{t} e^{i t \omega_{l n_{0}}(l)} \mathcal{A}_{n_{0}(l)}(\tau) \\
& \times e^{-i \delta \mathcal{E}_{l}(t, \tau)(t-\tau)} e^{-(1 / 2) \lambda_{l}(t, \tau)(t-\tau)} d \tau,
\end{aligned}
$$

where we define

$$
\begin{aligned}
\delta \mathcal{E}_{l}(t, \tau)= & \left\langle\phi_{l}^{(0)}|\hat{W}| \phi_{l}^{(0)}\right\rangle+\sum_{\substack{l^{\prime} \neq l, l^{\prime} \notin \Lambda_{s} \\
\mid \omega_{l l^{\prime}}<\Delta}} \frac{\left|\left\langle\phi_{l}^{(0)}|\hat{W}| \phi_{l^{\prime}}^{(0)}\right\rangle\right|^{2}}{\omega_{l l^{\prime}}} \\
& \times\left(1-\frac{\sin \omega_{l l^{\prime}} t-\sin \omega_{l l^{\prime}} \tau}{\omega_{l l^{\prime}}(t-\tau)}\right), \\
\lambda_{l}(t, \tau)= & 2 \sum_{\substack{l^{\prime} \neq l, l^{\prime} \notin \Lambda_{s} \\
\left|\omega_{l l^{\prime}}\right|<\Delta}}\left|\left\langle\phi_{l}^{(0)}|\hat{W}| \phi_{l^{\prime}}^{(0)}\right\rangle\right|^{2}\left(\frac{\cos \omega_{l l^{\prime}} t-\cos \omega_{l l^{\prime}} \tau}{\omega_{l l^{\prime}}^{2}(t-\tau)}\right) .
\end{aligned}
$$

Here, $n_{0}(l)$ refers to the optimized scar state that is spectrally closest to the $l$ th state, as illustrated in Fig. 8(b). These functions are straightforward generalizations of $\delta \mathcal{E}_{l}(t)$ and $\lambda_{l}(t)$ in Eq. (16). To get an intuitive sense of how the solution Eq. (21) behaves, we can momentarily take $\delta \mathcal{E}_{l}(t, \tau) \rightarrow \delta \overline{\mathcal{E}}_{l}$ and $\lambda_{l}(t, \tau) \rightarrow \bar{\lambda}_{l}$ to be constant since they are expected to be slowly varying. We similarly do this for $\delta \mathcal{E}_{n_{0}(l)}(t) \rightarrow \delta \overline{\mathcal{E}}_{n_{0}(l)}$ and $\lambda_{n_{0}(l)}(t) \rightarrow \bar{\lambda}_{n_{0}(l)}$. We can then perform the time integral in Eq. (21) explicitly to obtain

$$
\begin{aligned}
\mathcal{A}_{l \notin \Lambda_{s}}(t) \propto & \exp \left\{-i \delta \overline{\mathcal{E}}_{l} t-\frac{1}{2} \bar{\lambda}_{l} t\right\} \\
& -\exp \left\{-i\left(\delta \overline{\mathcal{E}}_{n_{0}(l)}-\omega_{l n_{0}(l)}\right) t-\frac{1}{2} \bar{\lambda}_{n_{0}(l)} t\right\} .
\end{aligned}
$$

This expression illustrates the overall behavior of the amplitudes $\mathcal{A}_{l \notin \Lambda_{s}}(t)$. Its absolute value vanishes at $t=0$ as well as at long times, reaching a maximum at intermediate times. The time scales in this evolution are the decay rate $\bar{\lambda}_{n_{0}(l)}$ of the optimized scar states that play the role of sources for the $J \neq 1$ towers; and the decay rate $\bar{\lambda}_{l}$ of the $J \neq 1$ towers themselves, which transition further to their spectral neighborhood. The balance between 
these two time scales determines the maximum value reached by $\left|\mathcal{A}_{l \notin \Lambda_{s}}(t)\right|$. Note from Eq (21) that this maximum value is also controlled by the random matrix element $\left\langle\phi_{l}^{(0)}|\hat{W}| \phi_{n_{0}(l)}^{(0)}\right\rangle$.

The final step we need to take is to perform the disorder average $\left[\mathcal{A}_{l^{\prime} \notin \Lambda_{s}}^{*}(t) \mathcal{A}_{l \notin \Lambda_{s}}(t)\right]$. Since it is somewhat more complicated and not very illuminating, we leave its explicit expression in Appendix C. Using this expression, we can then calculate the contribution to the magnetization from the $J \neq 1$ towers as

$$
\left.\left[\widetilde{\mathcal{M}}_{a}(\pi, t)\right]\right|_{\not_{S}} \approx \sum_{J \neq 1} \sum_{m=1}^{\mathcal{D}_{J}-1} 2 \operatorname{Re}\left(e^{i \Omega_{J m} t} \mathcal{Q}_{J m}(t) \Gamma_{J m}^{a,+1}\right) .
$$

In Fig. 9(b), we compare our explicit expression (red dots) with the numerically obtained contribution from the lower towers (yellow solid line), finding good agreement between them. The close match confirms our assumptions about the existence of lower towers as well as the manner in which they become populated. In Fig. 9(c) we add the analytic result when summed over all towers and again find good agreement with the numerical values for the full magnetization. Although it is more subtle in this case to infer that the $J \neq 1$ towers do not change the oscillation frequency, in Fig. 9(c) we see that the magnetization from lower towers continues to oscillate at a frequency close to the scar frequency.

Before continuing, it is worth stepping back to appreciate the reason the perturbative calculation worked so well. The simplicity of our perturbative approach hides the highly nontrivial nature of the stability of the oscillation frequency to disorder. Our treatment starts by assuming from the outset that the initial state (i.e. the $\mathbb{Z}_{2}$ state) populates a discrete set of states (i.e., the scar states), which are embedded in a dense spectrum. This is a highly unusual setup, which is the direct result of strong interactions in the underlying Hamiltonian. Thus, the essential insight is that the mechanism that makes the oscillation frequency robust is induced by a spectral structure that arises from strong interactions. That this structure is amenable to a perturbative treatment is a fortunate circumstance.

\section{Distribution and stability of scar resonances}

Our description of the disordered system suggests thinking about the PXP spectrum as being comprised of multiple towers of scars states that exhibit varying levels of ergodicity and entanglement. Upon disordering the system, these approximate scar states acquire a finite life time, making them effectively resonances in the disordered spectrum. To illustrate the formation of scar resonances in the system, we can calculate the distribution of the clean scar states in the basis of disordered eigenstates. In Fig. 10, we

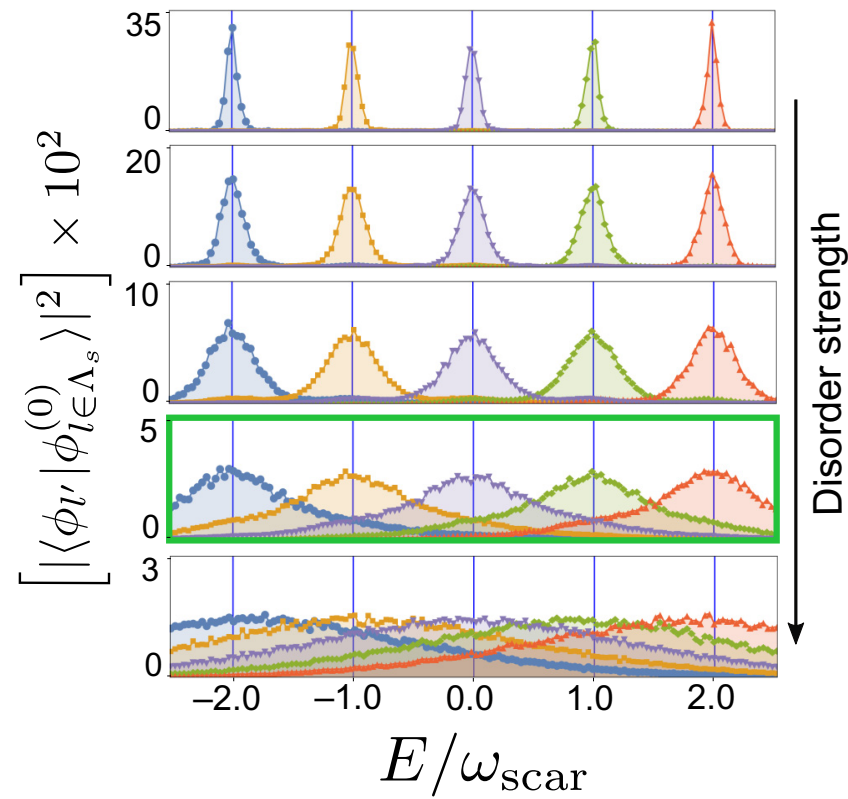

FIG. 10. Scar resonances in the spectrum. We plot the distribution of optimized scar states in the basis of disordered eigenstates for $L=12$ and disorder strengths for $W / \omega_{\text {scar }}=$ $0.10,0.19,0.34,0.63,0.17$ from top to bottom, respectively. To make it easier to visualize the shape of these distributions, we binned the energy axis and summed the probabilities inside each bin. As can be seen, for sufficiently weak disorder, the scar states are broadened in the disordered setting but remain distinguishable. Eventually, at a disorder strength $W_{c} \approx 0.63 \omega_{\text {scar }}$ (indicated here by the green frame), the width of these states increases so much that they can no longer be spectrally resolved.

show the disorder-averaged and coarse-grained probability $\left[\left|\left\langle\phi_{l} \mid \phi_{l \in \Lambda_{s}}^{(0)}\right\rangle\right|^{2}\right]$ as a function of the energy $E_{l}$ of the disordered eigenstate $\left|\phi_{l}\right\rangle$. To make it easier to visualize the overall shape of these distributions, we bin $\left|\left\langle\phi_{l} \mid \phi_{l \in \Lambda_{s}}^{(0)}\right\rangle\right|^{2}$ in discrete ranges of energy and sum them over each bin. For weak disorder relative to $W_{c}$, clear spectrally localized distributions are obtained that are centered at each of the scar energies of the clean limit. As the disorder strength is increased, their widths increase, which is what leads to the decay of the magnetization. Since their width is less than $\omega_{\text {scar }}$, the distribution corresponding to each scar state is clearly distinguished from each other. As long as the width of these resonances is small compared to the scar frequency, oscillations at the scar frequency will continue to be resolved in the dynamics. Importantly, the distributions remain centered at the scar energies, which is consistent with the rigidity in the oscillation frequency we found in Fig. 6(a), which we also concluded from our analytic result Eq. (17). When the disorder strength becomes sufficiently strong, the distributions overlap significantly (as illustrated by the green box in Fig. 10), and thus we expect oscillations at the scar frequency to disappear. 
That oscillations continue to be present for a time scale much greater than $\Omega^{-1}$ points to persistent deviations from ergodicity in the weakly disordered system, even though at long times the oscillations decay. This phenomenon is similar to what happens to the scar states of the undeformed and clean PXP model: most of them likely have a thermal component with respect to the scars of the optimized PXP model [70] (although some scar states, such as those found in Ref. [24] do not). The admixture of the thermal states into eigenstates is likely what leads to decay of observables such as magnetization. However, we would like to emphasize that the naive expectation is that nonconserved quantities at high energy densities should decay on the microscopic time scale, $\Omega^{-1}$. Long-lived excitations thus constitute a significant deviation from what is expected for nonintegrable systems, and is highly surprising, both theoretically and experimentally. Similar to the translationally invariant model distortion away from the optimized PXP, disorder provides a mechanism to control this time scale, which, as evidenced by our results, can significantly exceed $\Omega^{-1}$.

This picture of resonances allows us to infer an approximate value for $W_{c}$. Given that the contribution from $J \neq 1$ towers is smaller and appears at finite times, we focus on the main scar tower to determine this value. The width of these distributions in the frequency domain determines the decay of the scar amplitudes $\mathcal{A}_{l \in \Lambda_{s}}(t)$. As long as this broadening is sufficiently small, $\mathcal{A}_{l \in \Lambda_{s}}(t)$ will evolve slowly with respect to the scar period $2 \pi / \omega_{\text {scar }}$. When the disorder strength is increased, their widths continue to grow, up until when $W$ approaches $W_{c}$, at which point the distributions overlap significantly and are no longer distinguishable. We expect that $W_{c}$ is the disorder strength for which, after a time $T=2 \pi / \omega_{\text {scar }}$, the probability $\left|\mathcal{A}_{l \in \Lambda_{s}}(T)\right|^{2}=e^{-\lambda_{l}(T) T}$ is reduced to $1 / e$ of its value at $t=0$. The disorder averaged result, obtained from Eq. (18), leads to

$$
\frac{1}{\sqrt{\operatorname{det}\left(\mathbb{I}+\left(W_{c}^{2} / 6\right)\left\{G_{l \in \Lambda_{s}}^{\dagger}(T, 0)+G_{l \in \Lambda_{s}}(T, 0)\right\}\right)}} \sim e^{-1} .
$$

This produces the value $W_{c} \approx 0.34 \omega_{\text {scar }}$ for scar states near the middle of the spectrum for $L=14$, which is of the order of the value $W_{c} \approx 0.63 \omega_{\text {scar }}$ we found numerically in Fig. 7(b). We emphasize, however, that perturbation theory alone might not be able to account for the full physics at $W_{c}$. It is a subtle question whether there is a sharp transition between the scar region and the thermal phase. In fact, our diagram of dynamical regimes can serve as inspiration to experimentally explore the nature of the transition with present-day quantum simulators.

There is an important and subtle question regarding the scaling to the thermodynamic limit. Even though we found numerically that the magnetization of the system did not change appreciably with system size [see Fig. 7(a)], it was argued in Ref. [70] that perturbations that are added to a system with optimized scar states must inevitably thermalize for a sufficiently large system. Furthermore, using Lieb-Robinson bounds [70], it was argued that it would still be possible to observe nonergodic physics for a time scale $t^{*} \sim \mathcal{O}\left(\epsilon^{-1 / 2}\right)$, where $\epsilon$ is the strength of the perturbation. While in Ref. [70] the PXP Hamiltonian and the perturbation were clean systems, their argument has some bearing on our results. The reason that scars might be expected to thermalize in the disordered system is that matrix elements of the disorder operator between a scar state and another state that is ergodic must scale as $\mathcal{D}^{-1 / 2}$, whereas the density of states scales as $\mathcal{D}(\mathcal{D}$ being the dimension of the Hilbert space). This suggests that the factor $\lambda_{l}(t)$ in Eq. (16b) can potentially remain finite, so that nonergodic dynamics occurs for a time scale $t^{*} \sim \mathcal{O}\left(W^{-2}\right)$, consistent with Ref. [70]. However, higherorder terms in the perturbative calculation of the amplitude might get overwhelmed by the exponential growth of the density of states. Although we do not find numerical evidence of this in this work, we cannot rule out this possibility.

We do note, however, that the arguments in Ref. [70] assume that all states outside of the scar subspace are exactly ergodic. Instead, as we have found here, such states actually realize additional scar towers. The existence of this multitower structure will clearly impact the manner in which the scarred system thermalizes. Although these states are more highly entangled than the optimized scars, many of them are not completely volume-law states. This raises interesting questions about the nature of off-diagonal matrix elements of local observables. According to the ETH, these matrix elements must vanish with system size, which is contrary to what we found in the discussion leading to Fig. 4. Indeed, there are anomalously large matrix elements that contribute to the magnetization of the system and do not appear to decrease with system size, as evidenced by Fig. 7(a). This adds another subtle layer to the problem, and more broadly to questions of quantum thermalization in scarred systems, which warrants further investigation.

\section{SPATIOTEMPORAL CORRELATIONS}

Our quantitative perturbative description of the magnetization dynamics can also be extended to provide a physical understanding of spatiotemporal correlations starting from different initial states, such as clean scar eigenstates or the $\mathbb{Z}_{2}$ product state. It was shown in Ref. [65] that the equal-time connected correlator $\left\langle\phi_{n}^{(0)}\left|\left[\mathcal{P} \sigma_{r}^{z}(0) \mathcal{P}\right]\left[\mathcal{P} \sigma_{r+R}^{z}(0) \mathcal{P}\right]\right| \phi_{n}^{(0)}\right\rangle_{c}$ evaluated with respect to an optimized scar eigenstate converges to a nonzero value for large $R$, suggesting that the states are 
long-range spatially ordered. Furthermore, it can be argued that scars are temporally ordered as well [65]. We here consider the spatiotemporal correlator

$$
\mathcal{C}_{a}\left(r_{0}, R, t\right)=\left\langle\Psi\left|\left[\mathcal{P} \sigma_{r_{0}}^{a}(t) \mathcal{P}\right]\left[\mathcal{P} \sigma_{r_{0}+R}^{a}(0) \mathcal{P}\right]\right| \Psi\right\rangle_{c} .
$$

Temporal correlations can be explicitly calculated for the spatial Fourier transform $\widetilde{\mathcal{C}}_{a}(t)=\sum_{R, r_{0}=1}^{L} \int d t e^{i \pi R} \mathcal{C}_{a}$ $\left(r_{0}, r_{0}+R, t\right)$. Following the same steps as in the calculation of the magnetization, we find that (see Appendix D for details):

$$
\left[\widetilde{\mathcal{C}}_{a}(t)\right]=\mathcal{T}_{a}(t)-\left[\widetilde{\mathcal{M}}_{a}(\pi, t)\right] \widetilde{\mathcal{M}}_{a}(\pi, 0)
$$

where

$$
\begin{aligned}
\mathcal{T}_{a}(t) \approx & \sum_{J m} \sum_{s d}\left(\frac{\bar{\psi}_{n_{0}(J m-s d)}^{a} \Gamma_{J m-s d}^{a, s d}}{\psi_{n_{0}(J m)}}\right) \\
& \times\left[\mathcal{A}_{J m+s d}^{*}(t) \mathcal{A}_{J m}(t)\right] e^{i t \omega_{J m+s d, J m}}
\end{aligned}
$$

In this expression, we define $\bar{\psi}_{m}^{a}=\sum_{s, d} \psi_{J=1, m-s d} \Gamma_{1, m-s d}^{a, s d}$ with $s= \pm 1, d=1,3$. For convenience, in these sums we set $\psi_{J, m}=\Gamma_{J, m}^{a, s d}=0$ whenever $m$ is out of range in the sum. In contrast to the magnetization, for the calculation of temporal correlators we need to include matrix elements beyond the tridiagonal that are small but nevertheless contribute to the final temporal correlator. The expression Eq. (26) is completely determined, since we have already calculated $\left[\mathcal{A}_{l^{\prime}}^{*}(t) \mathcal{A}_{l}(t)\right]$ in previous sections. Similar to the magnetization, the dynamics of temporal correlators is thus also controlled by the existence of multitower scar resonances.

The expression Eq. (26) depends on both the choice of axis index $a$ of the Pauli operators, and the initial state $|\Psi\rangle$. As a first example, we calculate the correlator using the clean scar eigenstate at $E=0$ for magnetization component along $a=z$ axis. In Fig. 11 we show the contributions from the $J=1$ and $J \neq 1$ towers for both the numerical (dots) and analytic (solid lines) values, showing again very good agreement between both. The corresponding spatiotemporal correlator $\left[\left|\mathcal{C}_{z}\left(r_{0}, r_{0}+R, t\right)\right|\right]$ in the $(R, t)$ plane (with $r_{0}$ an odd site) underlying these results is shown in Fig. 11(a). The zero-energy scar state clearly leads to a periodic pattern in the $(R, t)$ plane indicating that spatially separated qubits are periodically correlated in time. While correlations are reduced by disorder as the temporal separation is increased, we have not observed decay as a function of $R$. This is counterintuitive, as disorder usually leads to a decay of spatial correlations with a characteristic length scale determined by the disorder strength. In this case, this decay appears to be absent for a fixed time, suggesting that spatial long-range order is maintained. This is quite remarkable, and deserves more detailed study

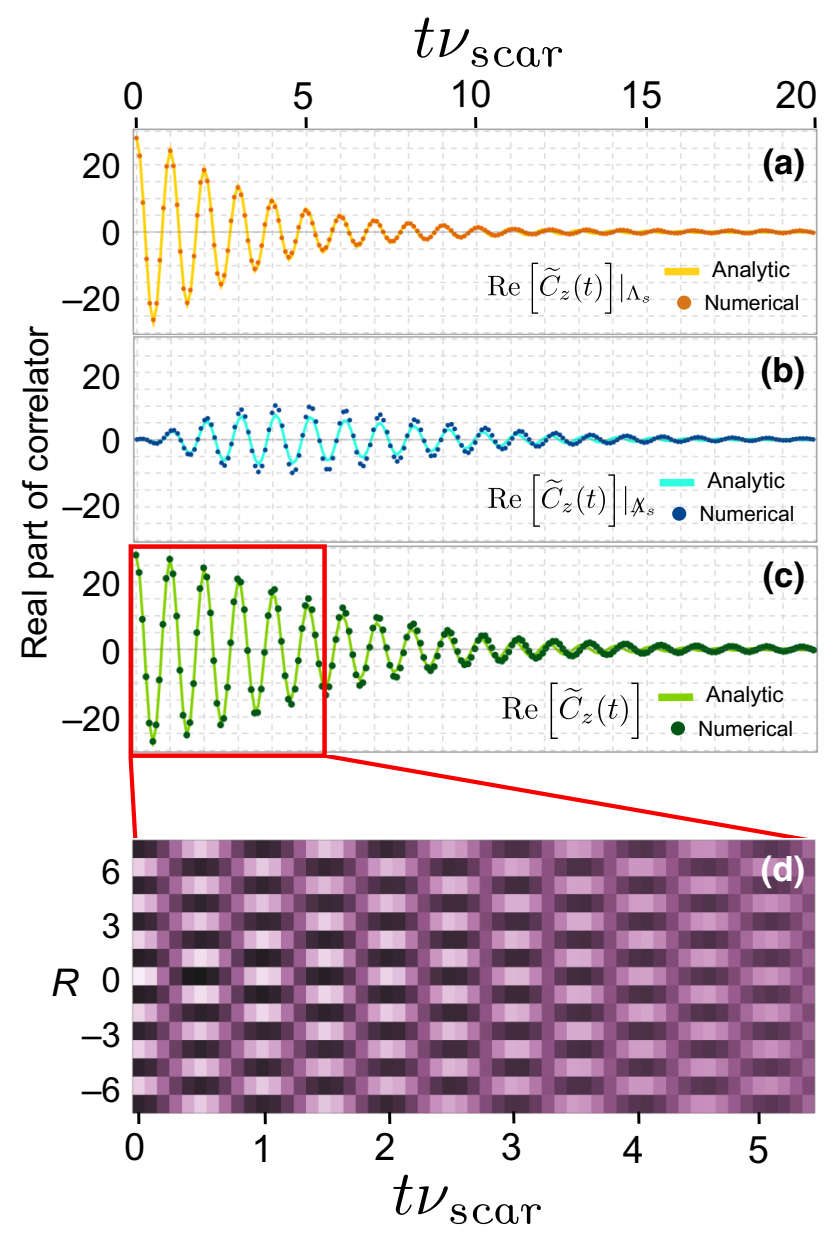

FIG. 11. Spatiotemporal correlations for $L=14$ and $W=$ $0.1 \omega_{\text {scar }}$ starting from clean scar eigenstate at zero energy as the initial state. The plots in (a)-(c) correspond to the different contributions to the net temporal correlator $\operatorname{Re}\left[\widetilde{C}_{z}(t)\right]$. The solid lines correspond to the analytic result Eq. (27) whereas the dots are numerically exact values. (d) Spatial resolution of the correlator $\left[\left|\mathcal{C}_{z}\left(r_{0}, r_{0}+R, t\right)\right|\right]$ (with $r_{0}$ an odd site) that underlies the oscillations found in the temporal correlator.

as a function of system size and disorder strength. It is also partially reminiscent of spatiotemporal correlations in time crystals, the nonequilibrium phases of matter that spontaneously break the time-translational symmetry of the Hamiltonian $[44,71,72]$. Time-translation symmetry is broken when $\lim _{R \rightarrow \infty} \mathcal{C}_{a}(r, R, t)$ oscillates with a frequency that differs from the temporal period of the Hamiltonian and is robust to small perturbations, signifying rigidity [54]. In the scar case, the Hamiltonian has full (in contrast to discrete) time-translation symmetry, and we indeed find evidence of spatial long-range order with a rigid frequency of oscillation that remains close to the clean scar frequency in spite of the presence of disorder. It is clear, nevertheless, that since disorder induces the decay of oscillations with time, the full analogy with time crystals is not complete.

While evaluating correlators starting with the scar eigenstates is illuminating, these states may not be easily 


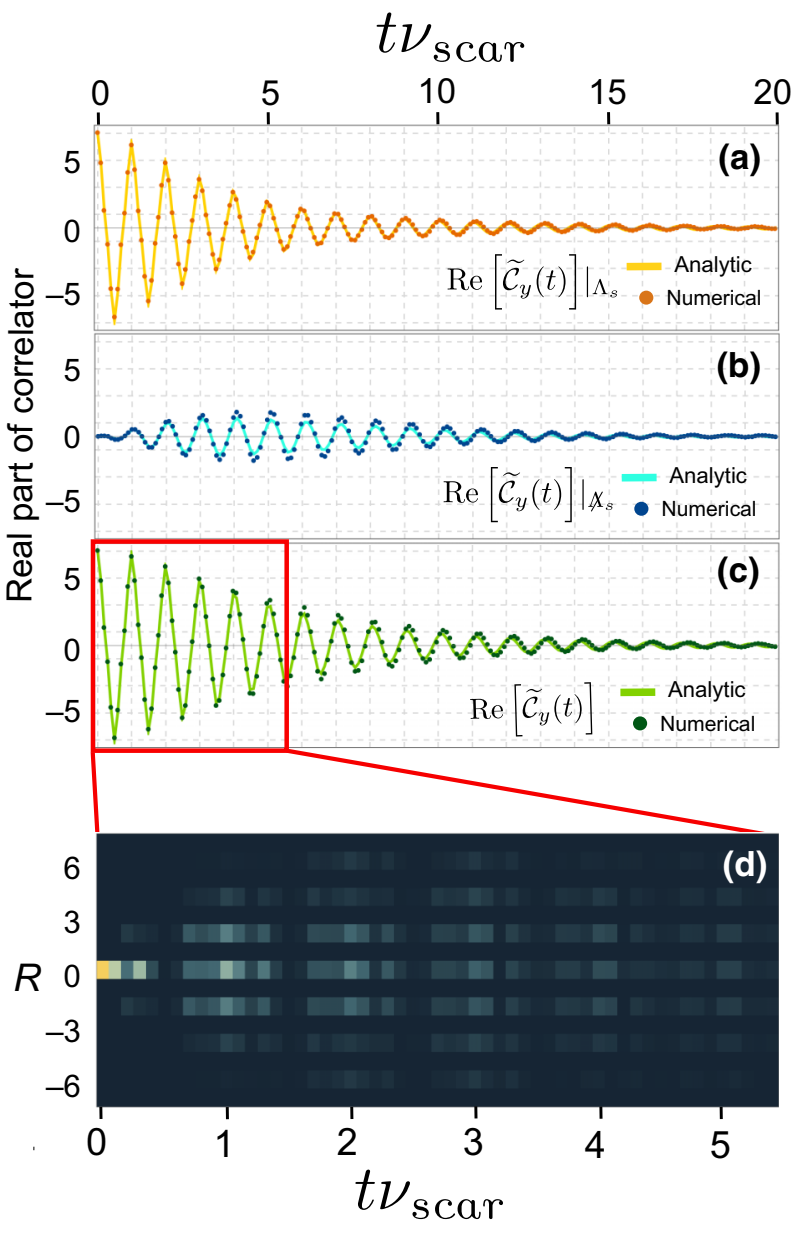

FIG. 12. Spatiotemporal correlations for $L=14$ and $W=$ $0.1 \omega_{\text {scar }}$ starting from the $|\Psi\rangle=\left|\mathbb{Z}_{2}\right\rangle$ state. The plots in (a)-(c) correspond to the different contributions to the temporal correlator $\operatorname{Re}\left[\widetilde{C}_{y}(t)\right]$. The solid lines correspond to the analytic result Eq. (27) whereas the dots are numerically exact values. (d) Spatial resolution of the correlator $\left[\left|\mathcal{C}_{y}\left(r_{0}, r_{0}+R, t\right)\right|\right]$ (with $r_{0}$ an odd site) that underlies the oscillations found in the temporal correlator.

accessible experimentally. For this reason, we also consider evaluating correlations starting from the $|\Psi\rangle=\left|\mathbb{Z}_{2}\right\rangle$ initial state. In this case, connected correlators with respect to the $\sigma_{r}^{z}$ operators vanish, so instead we analyze the $a=y$ component. In Fig. 12 we again show the contributions from the $J=1$ and $J \neq 1$ towers for both the numerical (dots) and analytic (solid lines) values, showing again very good agreement between both. Notably, although oscillations continue to occur at the scar frequency, the underlying spatiotemporal pattern is very different from the pattern that we obtained starting from a scar eigenstate. Now the correlation function begins concentrated at $R=0$, and spreads out as a function of time until it covers the full extent of the system. These correlations can in principle be probed in experiments. However, note that these results involve projected Pauli operators. To make even closer connection with future experiments, in the following section we evaluate them for the full interacting Hamiltonian without any projection operators.

\section{DIAGRAM OF DYNAMICAL REGIMES BETWEEN THE PARAMAGNETIC AND PXP LIMITS}

We now return to the full interacting Hamiltonian to examine the dynamics arising from scar resonances in the full parameter space $\left(W, J_{R}\right)$. This amounts to removing all projection operators in Eq. (6). In order to interpolate between the paramagnetic and PXP limits, we rescale the strength of the deformation coupling $\delta J_{R} \rightarrow\left(J_{R} / J_{\max }\right) \delta J_{R}$, where $J_{\max }=10^{2} \omega_{\mathrm{scar}}$ is the maximum value of the interaction strength we consider, which reaches very well into the PXP regime. Since the unprojected Hilbert space grows as $2^{L}$, we are confined in this section to smaller system sizes $L=8,10,12$. We focus on the connected temporal correlator

$$
\left[\widetilde{\mathcal{C}}_{y}^{\mathrm{Ryd}}(t)\right]=\left[\left\langle\mathbb{Z}_{2}\left|\widetilde{\sigma}_{\pi}^{y}(t) \widetilde{\sigma}_{\pi}^{y}(0)\right| \mathbb{Z}_{2}\right\rangle_{c}\right]
$$

This correlator differs from Eq. (26) by the absence of projection operators. This makes it a directly experimentally accessible quantity, although it has the downside that we can no longer evaluate it analytically. In Fig. 13(a), we show a contour plot of its Fourier transform $\left[\left|\widetilde{\mathcal{C}}_{y}^{\text {Ryd }}\left(\omega_{\text {scar }}\right)\right|\right]$ evaluated at the scar frequency in the $\left(W, J_{R}\right)$ parameter space. Oscillations at the scar frequency occur for interaction strengths greater than $J_{R} \approx \Omega$ and for disorder strengths up to around $W \sim W_{c}$. To pinpoint the boundary for this region, we again resort to the frequency participation parameter adapted to this correlation function $\bar{\Lambda}_{\omega}^{(q)}=\left[\int d \omega \mathcal{F}^{6}(\omega)\right]^{-1}$, where $\mathcal{F}(\omega)=(1 / \mathcal{N})\left[\left|\widetilde{\mathcal{C}}_{y}^{\text {Ryd }}(\omega)\right|\right]$ and $\mathcal{N}=\sqrt{\left.\int d \omega\left[\mid \widetilde{\mathcal{C}}_{y}^{\text {Ryd }}(\pi, \omega)\right]\right|^{2}}$. We find a line of parameters along which $\bar{\Lambda}_{\omega}^{(q)}$ reaches a maximum, as presented in show Fig. 13(a) with a dashed blue line. This boundary encompasses a region that is consistent with high values of $\left[\left|\widetilde{\mathcal{C}}_{y}^{\text {Ryd }}\left(\omega_{\text {scar }}\right)\right|\right]$. There is thus an ample regime of parameters for which it is possible to observe nonergodic dynamics, even for moderate interaction strengths and finite disorder. Interestingly, signatures of scar resonances are present even for low interactions when $J_{R} \sim \Omega$.

We further examine the ergodicity of the system using the mean level spacing ratio $\left[\left\langle r_{n}\right\rangle\right]$. Since the energy spectrum shifts significantly throughout the $\left(W, J_{R}\right)$ parameter space, we average over the spectral range $\left[\langle H\rangle_{\mathbb{Z}_{2}}-\delta E_{\mathbb{Z}_{2}},\langle H\rangle_{\mathbb{Z}_{2}}+\delta E_{\mathbb{Z}_{2}}\right]$. The energy spread $\delta E_{\mathbb{Z}_{2}}=$ $\sqrt{\left\langle H^{2}\right\rangle_{\mathbb{Z}_{2}}-\langle H\rangle_{\mathbb{Z}_{2}}^{2}}$ of the $\mathbb{Z}_{2}$ state with respect to the full Hamiltonian is the dynamically relevant part of the spectrum. In Fig. 13(b), we show a contour plot of $\left[\left\langle r_{n}\right\rangle\right]$ in the $\left(W, J_{R}\right)$ plane. A clear region is discernible for which $\left[\left\langle r_{n}\right\rangle\right]$ 

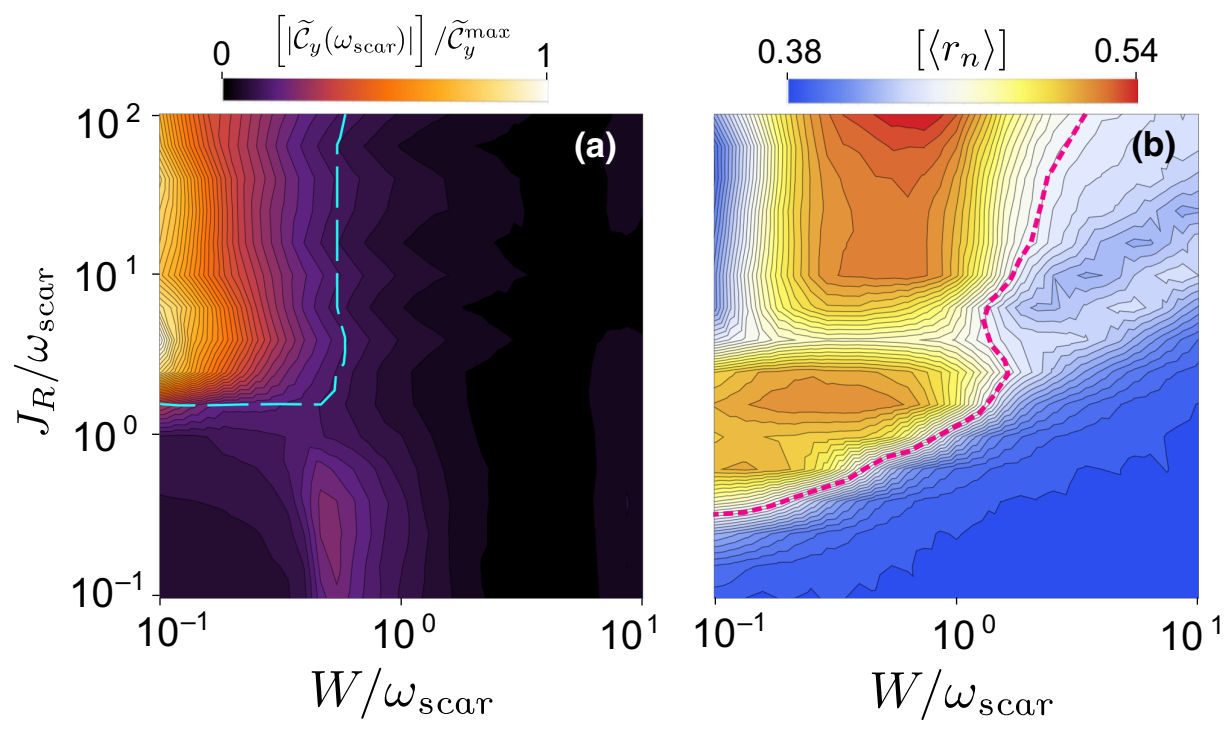

FIG. 13. (a) Contour plot of $\left.\left[\mid \widetilde{\mathcal{C}}_{y}^{\text {Ryd }}\left(\omega_{\text {scar }}\right)\right]\right], \quad$ normalized by the maximum value $\widetilde{C}_{y}^{\max }$ in this parameter space. The dashed line corresponds to the location of the peak in $\bar{\Lambda}_{\omega}^{(q=6)}$ for $L=12$. (b) Contour plot of the mean energy-level spacing ratio $\left[\left\langle r_{n}\right\rangle\right]$ that varies between the Poisson value $\left(\left[\left\langle r_{n}\right\rangle\right]=0.38\right)$ and the ergodic value $\left(\left[\left\langle r_{n}\right\rangle\right]=0.6\right)$. The dashed line corresponds to approximately the value $\left[\left\langle r_{n}\right\rangle\right]=0.45$, which we use to estimate the boundary between thermal and the MBL phases. is close to the ergodic value. A rough boundary for this region can be estimated to occur at the contour $\left[\left\langle r_{n}\right\rangle\right] \sim$ 0.45, which is the approximate value obtained in Fig. 3, and is shown by a magenta dashed in line in Fig. 13(b). The value of $\left[\left\langle r_{n}\right\rangle\right]$ generally increases to the left of this line as the system size is increased through $L=8,10,12$, and decreases to the right of this line. We note that due to the limited system sizes, there are some parameter points for which it is difficult to clearly discern a direction of flow for $\left[\left\langle r_{n}\right\rangle\right]$ as a function of $L$. Notwithstanding this, the boundary $\left[\left\langle r_{n}\right\rangle\right] \sim 0.45$ is consistent at strong interactions with the transition at the disorder strength $W_{\text {Th-L }}$ we found for the PXP model. It is also consistent with the weakly interacting limit, where $\left[\left\langle r_{n}\right\rangle\right]$ indeed is very close to the Poisson value, as expected for a MBL phase, which corresponds to a weakly interacting disordered paramagnet that is expected to be localized. Note that, similar to what we found in Fig. 3, there is a region to the right of the ergodic boundary for which, even though $\left[\left\langle r_{n}\right\rangle\right]$ flows to lower values as a function of system size, it decreases very slowly. This is consistent with the constrained MBL phase we found in the PXP limit.

Overall, it is clear that the ergodic region in Fig. 13(b) is larger than the region of scar resonances in Fig. 13(a). We thus obtain the overall diagram of dynamical regimes presented in Fig. 1(a). There are four regimes as a function of disorder and interaction strengths: a regime with scars resonances, another with a fully ergodic spectrum, a constrained MBL phase, and finally a MBL phase without kinematic constraints. Of note is an unexpected behavior within the ergodic region around $J_{R} \approx 4 \omega_{\text {scar }}$, where $\left[\left\langle r_{n}\right\rangle\right]$ appears to indicate weak ergodicity. Notably, there is a slight improvement in the strength of oscillations at the scar frequency in Fig. 13(a) in this region, perhaps suggesting that the weaker ergodicity is less detrimental for the existence of anomalous dynamics for these interaction strengths.

\section{DETECTION OF SCAR RESONANCES IN SIMULATORS}

A natural system where quantum scar resonances can be probed is the Rydberg simulator used to originally discover scar states. In this system, scars were observed for $J_{R} \sim 2 \pi \times 24 \mathrm{MHz}$ and $\Omega \sim 2 \pi \times 2 \mathrm{MHz}$ [4]. As a result $\omega_{\text {scar }} \sim 2 \pi \times 1.3 \mathrm{MHz}$ and $J_{R} \approx 19 \omega_{\text {scar }}$, which places this experimental system well within the regime that has scar eigenstates along the $W=0$ line in Fig. 1(a). The type of Zeeman disorder we considered can be implemented using drives with spatially varying Rabi frequencies. This platform should thus make it possible to observe the changes in dynamical regimes that arise both as a function of disorder as well as interaction strengths.

Superconducting qubit systems constitute another possible quantum simulator in which to study scar resonances. In charge qubit systems, for example, it is possible to realize the model [73]

$$
H=\sum_{r=1}^{L}\left(\frac{\Omega_{z}}{2} \tau_{r}^{z}+\frac{\Omega_{x}}{2} \tau_{r}^{x}+J_{x x} \tau_{r}^{x} \tau_{r+1}^{x}\right),
$$

which constitutes the transverse Ising model in the presence of a longitudinal field. The $\tau_{r}^{a}$ are Pauli matrices that act on the superconducting qubit charge states $\{|0\rangle,|1\rangle\}$. Up to a constant term and a unitary rotation, this model is equivalent to the Hamiltonian Eq. (1) when $\Omega_{x}=4 J_{x x}=$ $J_{R}$, which is what leads to the kinematic constraint in the system. In order to reach the regime of scar resonances, according to the diagram in Fig. 1(a), we must further have $\Omega_{z}<J_{x x}$. In these systems, it is possible to reach $J_{x x} \sim 2 \pi \times 100 \mathrm{MHz}$, as well as have a flux-tunable qubit frequency $\Omega_{z}$ [73], so reaching the scar resonance regime is feasible. Interestingly, Ising-type models of the form Eq. (29) can be realized in trapped ions as well [74]. However, an inevitable new ingredient in such a system is the 
presence of power-law Ising interactions [74-76]. Powerlaw interactions can lead to nontrivial dynamics that need to be examined more closely in the context of quantum many-body scars since they could lead to qualitative changes in the diagram Fig. 1(a).

Finally, one could alternatively probe the effects of disorder in scar systems with digital quantum simulators, although there are some challenges for currently available hardware [77]. However, there has been significant progress in the fidelity of single- and two-qubit gates in both ion traps [78,79] and SC qubits [80]. Furthermore, there has been progress in algorithms for quantum simulation beyond the coherence time of quantum devices [81]. As a result, it might be possible to access the physics of scar resonances in some devices in the near future. Interestingly, in addition to having the potential to model the Hamiltonian Eq. (1), digital simulators can directly simulate the strongly interacting limit described by the PXP model using Toffoli gates that effectively impose the kinematic constraint on sets of three contiguous qubits.

It is interesting to consider applications of quantum scar resonances if they are indeed implemented in any of these quantum simulators. For example, one can consider leveraging scar resonances as quantum sensors of the level of disorder in a given system and using that information to calibrate the underlying Hamiltonian. To be concrete, we envision the experimental measurement of the magnetization $\widetilde{\mathcal{M}}_{z}(\pi, t)$ as a function of time starting from the $\mathbb{Z}_{2}$ state. Its Fourier transform will reveal the broadened peak we found in Fig. 7(a), at $\omega=\omega_{\text {scar }}$ with a width $\Delta \omega$. Using our theoretical description, the width $\Delta \omega$ can be used to infer the value of the disorder strength $W$. The associated sensitivity $\Delta W$ of the measured disorder strength, a fundamental quantity that characterizes quantum sensors, can be straightforwardly estimated through the method of moments $[82,83]$. Interestingly, the sensitivity $\Delta W$ could be further controlled potentially by the use of squeezed initial states, not only because their entanglement has been shown to improve the sensitivity of quantum sensors, but also because such initial states can enable targeted access to different scar towers in the spectrum. This approach to quantum sensing is different from that proposed in Ref. [84] for clean scarred systems, as in our case we are directly exploiting the presence and structure of scar resonances.

Detecting the level of disorder can be particularly important in SC qubits systems that implement disorder on purpose to be able to, for example, operate cross-resonance gates [85]. It has been recently shown, in fact, that such systems are dangerously close to chaotic dynamics that can hinder the processing of quantum information [42]. Measuring the level of disorder in trapped-ion quantum simulators is also relevant, given its success in simulating time crystals and many-body localized phases that are stabilized by disorder $[43,44]$. Scar resonances could thus serve as another element in the tool box to calibrate quantum hardware, especially as quantum machines continue to be scaled up and holistic diagnostics are needed to characterize them. This constitutes one example of the possible applications of quantum many-body scars. As we mentioned previously, systems that avoid thermalization can play key roles in the development of quantum technologies and the results presented here can be used and expanded upon to explore these possibilities.

\section{CONCLUSIONS}

In this work, we studied the impact of disorder on systems with quantum many-body scars. We found that scar eigenstates become resonances that have a finite life time and remain centered at the scar energies of the clean system. As a result, observables exhibit nonergodic oscillations at the same scar frequency of the clean limit for a time scale $t^{*} \sim \mathcal{O}\left(W^{-2}\right)$. We confirmed this picture of scar resonances by calculating explicitly the disorder-averaged magnetization and temporal correlators of the system, which match closely with the values obtained numerically. We further examined the range of interaction and disorder strengths for which scar resonances are present in the system, and mapped out a diagram of other dynamical regimes that are accessed when scar resonances are lost at strong disorder and weak interactions.

The results presented in this work invite further investigation into the nonergodic nature of scar systems along several directions. For example, the rigidity we found in the spatiotemporal correlations of the PXP model poses intriguing questions regarding a possible framework for understanding quantum scar systems as realizations of continuous time-translation symmetry breaking in static (undriven) systems. It would also be interesting to explore further the transitions at strong disorder that eventually lead to the fully thermal and constrained MBL phases.

Finally, note that while our work has focused on the onedimensional model Eq. (6) as a case study, scar resonances can arise in other models and also in higher dimensions. This is clear from the derivation of Eq. (18), which did not make assumptions about the specific model at hand. The results in this work thus apply quite generally to other systems and consequently lay the groundwork to study other aspects of nonergodic dynamics arising from scar states in the presence of disorder.

\section{ACKNOWLEDGMENTS}

We are grateful to S. Kolkowitz for useful discussions. Research was supported by a QIS Award funded by U.S. Department of Energy, Office of Science, Basic Energy Sciences, at the University of Wisconsin under Award No. DE-SC0019449 and at Argonne National Laboratory. This work was performed in part at the Aspen Center for 
Physics, which is supported by National Science Foundation Grant No. PHY-1607611. We gratefully acknowledge the computing resources provided on Bebop, a highperformance computing cluster operated by the Laboratory Computing Resource Center at Argonne National Laboratory.

\section{APPENDIX A: MAGNETIZATION DYNAMICS IN THE CLEAN LIMIT}

In this Appendix, we derive the magnetization of the deformed PXP model. Since the $\mathbb{Z}_{2}$ state is approximately spanned by optimized scar states, we can write the initial state in the form $|\Psi(0)\rangle=\sum_{l \in \Lambda_{s}} \psi_{l}\left|\phi_{l}^{(0)}\right\rangle$, where $\Lambda_{s}$ the set of indices that label optimized scars and $\left\{\left|\phi_{l}^{(0)}\right\rangle\right\}$ is the set of eigenstates of the clean system. Since the timeevolved state is given by $|\Psi(t)\rangle=\sum_{l \in \Lambda_{s}} e^{-i E_{l}^{(0)} t} \psi_{l}\left|\phi_{l}^{(0)}\right\rangle$, the magnetization takes the form

$$
\widetilde{\mathcal{M}}_{a}(\pi, t)=\sum_{l, l^{\prime} \in \Lambda_{s}} e^{i \omega_{l^{\prime}} t^{t}} \psi_{l^{\prime}}^{*} \psi_{l}\left\langle\phi_{l^{\prime}}^{(0)}\left|\widetilde{\sigma}_{\pi}^{a}\right| \phi_{l}^{(0)}\right\rangle,
$$

where we define $\omega_{l^{\prime} l}=E_{l^{\prime}}^{(0)}-E_{l}^{(0)}$. Thus, the problem reduces to determining the functional form of $\left\langle\phi_{l^{\prime}}^{(0)}\left|\widetilde{\sigma}_{\pi}^{a}\right| \phi_{l}^{(0)}\right\rangle$. As was found in Ref. [30], angularmomentum operators can be defined within the scar subspace as

$$
\begin{aligned}
S_{x} & =\frac{1}{2 \eta} \sum_{r} \mathcal{P}_{s}\left(\sigma_{r}^{x}+\delta \sigma_{r}^{x}\right) \mathcal{P}_{s}, \\
S_{y} & =\frac{1}{2 \eta} \sum_{r}(-1)^{r} \mathcal{P}_{s}\left(\sigma_{r}^{y}+\delta \sigma_{r}^{y}\right) \mathcal{P}_{s}, \\
S_{z} & =-i\left[S_{x}, S_{y}\right],
\end{aligned}
$$

where $\mathcal{P}_{s}=\sum_{l \in \Lambda_{s}}\left|\phi_{l}^{(0)}\right\rangle\left\langle\phi_{l}^{(0)}\right|$ is the projector into the subspace of optimized scars, and $\delta \sigma_{r}^{a}=\left(2 \delta J_{R} / \Omega\right)$ $\left(\sigma_{r+2}^{z}+\sigma_{r-2}^{z}\right) \sigma_{r}^{a}$. These operators approximately satisfy angular-momentum commutation relations within the subspace of scar states, so that the $L+1$ states span the tower of maximum total angular momentum $S=L / 2$. Now, note that $S_{x}$ and $S_{y}$ are slight deformations of the operators $\sigma_{0}^{x}$ and $\sigma_{\pi}^{y}$, respectively, since $\delta J_{R} / \Omega$ is small. As a result, we approximate

$$
\begin{aligned}
& \left\langle\phi_{l \in \Lambda_{s}}^{(0)}\left|S_{x}\right| \phi_{l^{\prime} \in \Lambda_{s}}^{(0)}\right\rangle \approx \frac{1}{2 \eta}\left\langle\phi_{l \in \Lambda_{s}}^{(0)}\left|\widetilde{\sigma}_{0}^{x}\right| \phi_{l^{\prime} \in \Lambda_{s}}^{(0)}\right\rangle, \\
& \left\langle\phi_{l \in \Lambda_{s}}^{(0)}\left|S_{y}\right| \phi_{l^{\prime} \in \Lambda_{s}}^{(0)}\right\rangle \approx \frac{1}{2 \eta}\left\langle\phi_{l \in \Lambda_{s}}^{(0)}\left|\widetilde{\sigma}_{\pi}^{y}\right| \phi_{l^{\prime} \in \Lambda_{s}}^{(0)}\right\rangle .
\end{aligned}
$$

We emphasize that, while we have disregarded the deformation term proportional to $\delta J_{R} / \Omega$ in these angularmomentum operators, the eigenstates $\left\{\left|\phi_{l}^{(0)}\right\rangle\right\}$ are obtained with the deformed Hamiltonian. This reduces the deviation from the ideal $\mathfrak{s u}(2)$ algebra found in Ref. [30].

Next, the optimization of scar states implemented in Ref. [30] is designed so that the $L+1$ states $\left\{\left|K_{n}\right\rangle\right\}$ obtained by the forward scattering approximation (FSA) proposed in Ref. [6] become exact eigenstates of $S_{z}$ so that $\left\langle K_{n}\left|S_{z}\right| K_{n^{\prime}}\right\rangle \approx m_{n} \delta_{n n^{\prime}}$, where $m_{n}=-L / 2, \ldots, L / 2$. As a result, the projection operator into the scar subspace can be written in the unitarily equivalent form $\mathcal{P}_{s}=\sum_{n}\left|K_{n}\right\rangle\left\langle K_{n}\right|$, since the $\left\{\left|K_{n}\right\rangle\right\}$ must span this subspace. These FSA states are constructed by repeated application of the operator $H^{+}=\sum_{r}\left(\sigma_{r}^{x}+\delta \sigma_{r}^{x}\right)+i \sum_{r}(-1)^{r}\left(\sigma_{r}^{y}+\delta \sigma_{r}^{y}\right)$ on the $\mathbb{Z}_{2}$ state [30]. Since $\left[\sigma_{\pi}^{z}, H^{+}\right]=2 H^{+}$and $\left|\mathbb{Z}_{2}\right\rangle$ is an eigenstate of $\sigma_{\pi}^{z}$, then $\left\langle K_{n}\left|\sigma_{\pi}^{z}\right| K_{n^{\prime}}\right\rangle=2 m_{n} \delta_{n n^{\prime}}$. Thus, we can write approximately

$$
S_{z} \approx \frac{1}{2} \mathcal{P}_{s} \widetilde{\sigma}_{\pi}^{z} \mathcal{P}_{s}
$$

Having established the approximate $\mathfrak{s u}(2)$ algebra satisfied by $\sigma_{0}^{x}$ and $\sigma_{\pi}^{y, z}$, we now express the latter in the form

$$
\begin{aligned}
& \mathcal{P}_{s} \widetilde{\sigma}_{\pi}^{z} \mathcal{P}_{s}=\mathcal{P}_{s}\left(\sigma_{s}^{+}+\sigma_{s}^{-}\right) \mathcal{P}_{s}, \\
& \mathcal{P}_{s} \widetilde{\sigma}_{\pi}^{y} \mathcal{P}_{s}=\eta \mathcal{P}_{s}\left(\sigma_{s}^{+}-\sigma_{s}^{-}\right) \mathcal{P}_{s} .
\end{aligned}
$$

Here, the $\tilde{\sigma}_{s}^{ \pm}$act as ladder operators on the scar eigenstates: $\left\langle\phi_{l^{\prime} \in \Lambda_{s}}^{(0)}\left|\sigma_{s}^{ \pm}\right| \phi_{l \in \Lambda_{s}}^{(0)}\right\rangle \propto \delta_{l^{\prime}, l_{ \pm 1}}$ where the notation $l_{ \pm 1}$ refers to the index of the scar state that is spectrally separated by $\pm \omega_{\text {scar }}$ from the $l$ th scar state. Thus, the matrix elements $\left\langle\phi_{l^{\prime} \in \Lambda_{s}}^{(0)}\left|\widetilde{\sigma}_{\pi}^{a}\right| \phi_{l \in \Lambda_{s}}^{(0)}\right\rangle$ precisely pick out the phases that oscillate at the frequency $\omega_{l_{ \pm}}=\omega_{\text {scar }}$. We then obtain the magnetization components

$$
\begin{aligned}
\widetilde{\mathcal{M}}_{y}(\pi, t) & \approx \eta L \sin \left(\omega_{\text {scar }} t\right), \\
\widetilde{\mathcal{M}}_{z}(\pi, t) & \approx-L \cos \left(\omega_{\text {scar }} t\right),
\end{aligned}
$$

To arrive at this expression, we have used that $\sum_{l \in \Lambda_{s}^{\prime}} 2 \operatorname{Re}\left(\psi_{l+1}^{*} \psi_{l}\left\langle\phi_{l_{+1}}^{(0)}\left|\sigma_{s}^{+}\right| \phi_{l}^{(0)}\right\rangle\right) \approx \widetilde{\mathcal{M}}_{z}(\pi, 0)=-L$, where $\Lambda_{s}^{\prime}$ excludes the highest-energy scar state. These are the expressions presented in Eq. (13) of the main text.

\section{APPENDIX B: REORDERING OF BASIS}

In this Appendix, we discuss how to reorder the energy eigenstates into towers. Suppose we take an energy eigenstate $\left|\phi_{m_{1}}^{(0)}\right\rangle$ from the bottom of the spectrum and we apply the ladder operator once. We use the operators defined in the main text

$$
\sigma_{s}^{ \pm}=\frac{1}{2}\left(\widetilde{\sigma}_{\pi}^{z} \mp i \eta^{-1} \widetilde{\sigma}_{\pi}^{y}\right) .
$$

As we discussed in Appendix A1, these are approximate ladder operators within the space of optimized scar states. 
Here, however, we examine their behavior in the full PXP Hilbert space. In general, applying $\sigma_{s}^{+}$on any given state can result in a linear combination over all eigenstates of the clean system. However, numerically one finds that this linear combination is highly concentrated at a particular state $\left|\phi_{m_{2}}^{(0)}\right\rangle$ of higher energy than $\left|\phi_{m_{1}}^{(0)}\right\rangle$. If we subsequently compute $\sigma_{s}^{+}\left|\phi_{m_{2}}^{(0)}\right\rangle$, one again finds that this state is largely peaked at another eigenstate $\left|\phi_{m_{3}}^{(0)}\right\rangle$ with higher energy than $\left|\phi_{m_{1}}^{(0)}\right\rangle$ and $\left|\phi_{m_{2}}^{(0)}\right\rangle$. By repeating this procedure, we eventually arrive at a state $\left|\phi_{m_{\max }}^{(0)}\right\rangle$ for which the further application of the operator does not produce a peaked distribution. Thus, $\sigma_{s}^{+}$acts like a ladder operator for the set of states $\left\{\left|\phi_{m_{i}}^{(0)}\right\rangle\right\}$, even in cases where the states do not belong to the scar subspace. By iteratively applying this procedure to all eigenstates, we can systematically group all energy eigenstates into sets $\left\{\left|\phi_{J m}^{(0)}\right\rangle\right\}$, where $J$ labels the set and $m=1, \ldots, \mathcal{D}_{J}$ labels the state within that set. In this notation, we can include the scar space in the set $J=1$, since this set is generated by starting with the ground state. We found that a small number of states do not have strong matrix elements with any other state in the basis, so they form sets by themselves according to our algorithm. Clearly, such states do not contribute to the magnetization.

This is the set of steps we took to reorder the basis of energy eigenstates. For example, the case $L=8$ presented in the main text leads to 13 towers of sizes: $\mathcal{D}_{1}=9, \mathcal{D}_{2}=$ $\mathcal{D}_{3}=7, \mathcal{D}_{4}=\mathcal{D}_{5}=6, \mathcal{D}_{7-13}=1$.

\section{APPENDIX C: DISORDER-AVERAGED AMPLITUDE DYNAMICS}

In this section, we calculate the disorder-averaged quantity $\left[\mathcal{A}_{l^{\prime}}^{*}(t) \mathcal{A}_{l}(t)\right]$, where the amplitudes $\mathcal{A}_{l}(t)$ determine the time evolution of the system $|\Psi(t)\rangle=$ $\sum_{l} \mathcal{A}_{l}(t) e^{-i E_{l}^{(0)} t}\left|\phi_{l}^{(0)}\right\rangle$, where $\left|\phi_{l}^{(0)}\right\rangle$ is the $l$ th eigenstate of the clean Hamiltonian with energy $E_{l}^{(0)}$. The Schrodinger equation for these amplitudes is given by

$$
i \frac{d}{d t} \mathcal{A}_{l}(t)=\sum_{l^{\prime}}\left\langle\phi_{l}^{(0)}|\hat{W}| \phi_{l^{\prime}}^{(0)}\right\rangle \mathcal{A}_{l^{\prime}}(t) e^{i \omega_{l l^{\prime}}}
$$

where $\omega_{l l^{\prime}}=E_{l}^{(0)}-E_{l^{\prime}}^{(0)}$. We proceed by first calculating the dynamics of the amplitudes corresponding to optimized scars $l \in \Lambda_{s}$ and afterwards obtain the dynamics of the remaining amplitudes $l \notin \Lambda_{s}$.

\section{Dynamics of the tower of optimized scars}

We begin by calculating the dynamics of the tower of maximum angular momentum, which consists of the optimized scar states:

$$
i \frac{d}{d t} \mathcal{A}_{l \in \Lambda_{s}}(t)=\sum_{l^{\prime}}\left\langle\phi_{l}^{(0)}|\hat{W}| \phi_{l^{\prime}}^{(0)}\right\rangle e^{i \omega_{l l^{\prime}} t} \mathcal{A}_{l^{\prime}}(t) .
$$

The right-hand side of this equation in general includes all possible states in the Hilbert space. However, for weak disorder, we expect that only a subset of states in the spectral neighborhood of the state $\left|\phi_{l \in \Lambda_{s}}^{(0)}\right\rangle$ will be relevant in the dynamics of $\mathcal{A}_{l \in \Lambda_{s}}(t)$. We thus truncate the sum to obtain

$$
\begin{aligned}
i \frac{d}{d t} \mathcal{A}_{l \in \Lambda_{s}}(t) \approx & \left\langle\phi_{l}^{(0)}|\hat{W}| \phi_{l}^{(0)}\right\rangle \mathcal{A}_{l}(t) \\
& +\sum_{\substack{l^{\prime} \notin \Lambda_{s} \\
\left|\omega_{l l^{\prime}}\right|<\Delta}}\left\langle\phi_{l}^{(0)}|\hat{W}| \phi_{l^{\prime}}^{(0)}\right\rangle e^{i \omega_{l l^{\prime}}{ }^{t}} \mathcal{A}_{l^{\prime}}(t) .
\end{aligned}
$$

Given that the number of states in the spectral vicinity of the $l$ 'th optimized scar state grows exponentially in the system size, we expect that $\mathcal{A}_{l \in \Lambda_{s}}(t)$ will decay into those states irreversibly. The amplitudes to which the initial state decays are themselves ruled by the equation

$$
i \frac{d}{d t} \mathcal{A}_{l \notin \Lambda_{s}}(t)=\sum_{\left|\omega_{l l^{\prime}}\right|<\Delta}\left\langle\phi_{l}^{(0)}|\hat{W}| \phi_{l^{\prime}}^{(0)}\right\rangle \mathcal{A}_{l^{\prime}}(t) e^{i \omega_{l l^{\prime}} t}
$$

which can be written in the integral form

$$
\mathcal{A}_{l \notin \Lambda_{s}}(t)=(-i) \int_{0}^{t} d \tau \sum_{\left|\omega_{l l^{\prime}}\right|<\Delta}\left\langle\phi_{l}^{(0)}|\hat{W}| \phi_{l^{\prime}}^{(0)}\right\rangle \mathcal{A}_{l^{\prime}}(\tau) e^{i \omega_{l l^{\prime}} \tau} .
$$

When this is plugged into the equation for $\mathcal{A}_{l \in \Lambda_{s}}(t)$, we obtain

$$
\begin{aligned}
i \frac{d}{d t} \mathcal{A}_{l \in \Lambda_{s}}(t) & \approx\left\langle\phi_{l}^{(0)}|\hat{W}| \phi_{l}^{(0)}\right\rangle \mathcal{A}_{l}(t)-i \sum_{\substack{l^{\prime} \notin \Lambda_{s} \\
\left|\omega_{l l^{\prime}}\right|<\Delta}}\left\langle\phi_{l}^{(0)}|\hat{W}| \phi_{l^{\prime}}^{(0)}\right\rangle e^{i \omega_{l l^{\prime}} t} \int_{0}^{t} d \tau \sum_{\left|\omega_{l^{\prime} l^{\prime}}\right|<\Delta}\left\langle\phi_{l^{\prime}}^{(0)}|\hat{W}| \phi_{l^{\prime \prime}}^{(0)}\right\rangle \mathcal{A}_{l^{\prime \prime}}(\tau) e^{i \omega_{l^{\prime} l^{\prime \prime}} \tau}, \\
& \approx\left\langle\phi_{l}^{(0)}|\hat{W}| \phi_{l}^{(0)}\right\rangle \mathcal{A}_{l}(t)-i \int_{0}^{t} d \tau\left\{\sum_{\substack{l^{\prime} \notin \Lambda_{s} \\
\left|\omega_{l l^{\prime}}\right|<\Delta}}\left|\left\langle\phi_{l}^{(0)}|\hat{W}| \phi_{l^{\prime}}^{(0)}\right\rangle\right|^{2} e^{-i \omega_{l l^{\prime}}(\tau-t)}\right\} \mathcal{A}_{l}(\tau),
\end{aligned}
$$




$$
\begin{aligned}
& \approx\left\langle\phi_{l}^{(0)}|\hat{W}| \phi_{l}^{(0)}\right\rangle \mathcal{A}_{l}(t)-i \mathcal{A}_{l}(t) \int_{0}^{t} d \tau\left\{\sum_{\substack{l^{\prime} \notin \Lambda_{s} \\
\left|\omega_{l l^{\prime}}\right|<\Delta}}\left|\left\langle\phi_{l}^{(0)}|\hat{W}| \phi_{l^{\prime}}^{(0)}\right\rangle\right|^{2} e^{-i \omega_{l l^{\prime}}(\tau-t)}\right\}, \\
& =z_{l \in \Lambda_{s}}(t) \mathcal{A}_{l \in \Lambda_{s}}(t),
\end{aligned}
$$

where $\quad z_{l \in \Lambda_{s}}(t)=\left\langle\phi_{l}^{(0)}|\hat{W}| \phi_{l}^{(0)}\right\rangle-i \int_{0}^{t} d \tau \sum_{\substack{l^{\prime} \notin \Lambda_{s} \\\left|\omega_{l l^{\prime}}\right|<\Delta}}$ $\left|\left\langle\phi_{l}^{(0)}|\hat{W}| \phi_{l^{\prime}}^{(0)}\right\rangle\right|^{2} e^{-i \omega_{l l^{\prime}}(\tau-t)}$. To obtain Eq. (C7), we made two approximations, which are typically used, for example, in the study of the decay of a discrete level into a continuum. The first approximation was made in going from Eq. (C4) to Eq. (C5), where we dropped matrix elements that do not connect states with the scar state $\left|\phi_{l \in \Lambda_{s}}^{(0)}\right\rangle$, as these are not dominant in the process of this initial state decaying irreversibly. The second approximation was made in going from Eq. (C5) to Eq. (C6), where we used that the factor in curly brackets is peaked at $t=\tau$, so we can evaluate it at $\mathcal{A}(\tau=t)$ and pull it out of the integral. We thus obtain an equation exclusively for $\mathcal{A}_{l \in \Lambda_{s}}(t)$, which leads to the solution

$$
\mathcal{A}_{l \in \Lambda_{s}}(t)=\psi_{l} e^{-i \int_{0}^{t} z_{l}\left(\tau^{\prime}\right) d \tau^{\prime}} .
$$

Now, in order to perform the disorder averaging, it is convenient to rewrite this so that the dependence on the random variables of the potential are made explicit. We define the vectors $\mathbf{h}^{T}=\left\{h_{a(1)}[r(1)], \ldots, h_{a(3 L)}[r(3 L)]\right\}$ and $\rho_{m n}^{T}=$ $\left\{\rho_{m n}^{1}, \ldots, \rho_{m n}^{3 L}\right\}$ where $\left(\rho_{m n}\right)_{l}=\left\langle\phi_{n}^{(0)}\left|\sigma_{r(l)}^{a(l)}\right| \phi_{n}^{(0)}\right\rangle$, with $l=$ $1, \ldots, 3 L, a(l)=(l-1) \bmod 3$, and $r(l)=\lfloor(l-1) / 3\rfloor$. The matrix elements of the disorder operator can then be written in the form

$$
\begin{aligned}
\left\langle\phi_{l}^{(0)}|\hat{W}| \phi_{l^{\prime}}^{(0)}\right\rangle & =\sum_{r a} h_{a}(r)\left\langle\phi_{l}^{(0)}\left|\sigma_{r}^{a}\right| \phi_{l^{\prime}}^{(0)}\right\rangle \\
& =\rho_{l l^{\prime}}^{T} \cdot \mathbf{h}, \\
\left|\left\langle\phi_{l}^{(0)}|\hat{W}| \phi_{l^{\prime}}^{(0)}\right\rangle\right|^{2} & =\left(\mathbf{h}^{T} \cdot \rho_{l l^{\prime}}^{*}\right)\left(\rho_{l l^{\prime}}^{T} \cdot \mathbf{h}\right) \\
= & \mathbf{h}^{T} \cdot\left(\rho_{l l^{\prime}}^{*} \cdot \rho_{l l^{\prime}}^{T}\right) \cdot \mathbf{h},
\end{aligned}
$$

so we finally have the expression

$$
\int_{0}^{t} z_{l \in \Lambda_{s}}(\tau) d \tau=t \rho_{l l}^{T} \cdot \mathbf{h}-i \mathbf{h}^{T} \cdot G_{l}(t, 0) \cdot \mathbf{h}
$$

where $G_{l \in \Lambda_{s}}\left(t_{1}, t_{2}\right)=\sum_{\substack{l \neq l^{\prime}, l^{\prime} \notin \Lambda_{s} \\\left|\omega_{l l^{\prime}}\right|<\Delta}}\left(\rho_{l l^{\prime}}^{*} \cdot \rho_{l l^{\prime}}^{T}\right) f_{l l^{\prime}}\left(t_{1}, t_{2}\right)$ and $f_{m n}\left(t_{1}, t_{2}\right)=i\left[\left(t_{1}-t_{2}\right) / \omega_{m n}\right]+\left(e^{i \omega_{m n} t_{2}}-e^{i \omega_{m n} t_{1}}\right) / \omega_{m n}^{2}$. We define this function in terms of two temporal arguments because it will arise in this form when we calculate the dynamics of the lower towers. The amplitude can then be written explicitly in the form

$$
\mathcal{A}_{l \in \Lambda_{S}}(t) \approx \psi_{l} e^{-i t \rho_{l l} \cdot \mathbf{h}} e^{-\mathbf{h}^{T} \cdot G_{l}(t, 0) \cdot \mathbf{h}} .
$$

With this expression at hand, we can calculate $\left[\mathcal{A}_{l^{\prime}}^{*}(t)\right.$ $\mathcal{A}_{l}(t)$ ]. To do this, we use the Gaussian distribution $P(\mathbf{h})=$ $\left[1 /\left(\pi W^{2} / 6\right)^{3 L / 2}\right] e^{-\left(6 / W^{2}\right) \mathbf{h}^{2}}$. This distribution has the same first and second moments of the box distribution we used numerically. We thus have

$$
\begin{aligned}
{\left[\mathcal{A}_{l^{\prime}}^{*}(t) \mathcal{A}_{l}(t)\right] \approx } & \frac{\psi_{l^{\prime}}^{*} \psi_{l}}{\left(\pi W^{2} / 6\right)^{3 L / 2}} \int_{\mathbb{R}^{3 L}} d \mathbf{h} e^{-\left(6 / W^{2}\right) \mathbf{h}^{2}} \\
& \times e^{i t\left(\rho_{l^{\prime} l^{\prime}}-\rho_{l l}\right) \cdot \mathbf{h}-\mathbf{h}^{T} \cdot\left[G_{l^{\prime}}^{*}(t, 0)+G_{l}(t, 0)\right] \cdot \mathbf{h}} \\
= & \psi_{l^{\prime}}^{*} \psi_{l} \sqrt{\operatorname{det}\left\{\alpha_{l^{\prime} l}(t)\right\}} \\
\times & e^{-\left(W^{2} t^{2} / 24\right)\left(\rho_{l^{\prime} l^{\prime}}-\rho_{l l}\right) \cdot \alpha_{l^{\prime}}(t) \cdot\left(\rho_{l^{\prime} l^{\prime}}-\rho_{l l}\right)}
\end{aligned}
$$

where $\alpha_{l^{\prime} l}(t)=\left\{\mathbb{I}+\left(W^{2} / 6\right)\left[G_{l^{\prime}}^{*}(t, 0)+G_{l}(t, 0)\right]\right\}^{-1}$. This is the expression Eq. (18) discussed in the main text.

\section{Dynamics of $\boldsymbol{J} \neq \mathbf{1}$ towers}

We now move on to examine the dynamics of the other towers. The main difference with the tower of optimized scars is the initial condition. Again, the equation is given by

$$
i \frac{d}{d t} \mathcal{A}_{l \notin \Lambda_{s}}(t)=\sum_{l^{\prime}}\left\langle\phi_{l}^{(0)}|\hat{W}| \phi_{l^{\prime}}^{(0)}\right\rangle A_{l^{\prime}}(t) e^{i \omega_{l l^{\prime}}}
$$

Once the amplitudes for the lower towers begin to grow from zero, there will be tunneling from these states to all other spectrally near-by states. The tower of optimized scars thus serves as a source for the lower towers and the spectrally surrounding states serve as a sink into which the amplitudes $\mathcal{A}_{l \notin \Lambda_{s}}(t)$ decay irreversibly. Similar to the approach used for the tower of maximal angular momentum, we again express the amplitude of the states to which the amplitude $\mathcal{A}_{l}(t)$ decays in the integral form $\mathcal{A}_{l^{\prime}}(t)=$ $(-i) \int_{0}^{t} d \tau \sum_{\left|\omega_{l^{\prime} l^{\prime \prime}}\right|<\Delta}\left\langle\phi_{l^{\prime}}^{(0)}|\hat{W}| \phi_{l^{\prime \prime}}^{(0)}\right\rangle \mathcal{A}_{l^{\prime \prime}}(\tau) e^{i \omega_{l^{\prime} l^{\prime \prime}} \tau}$. We can then write the equation for $\mathcal{A}_{l \notin \Lambda_{s}}(t)$ as 


$$
\begin{aligned}
& i \frac{d}{d t} \mathcal{A}_{l \notin \Lambda_{s}}(t)=\left\langle\phi_{l}^{(0)}|\hat{W}| \phi_{l}^{(0)}\right\rangle \mathcal{A}_{l}(t)+\left\langle\phi_{l}^{(0)}|\hat{W}| \phi_{n_{0}(l)}^{(0)}\right\rangle e^{i t \omega_{l n_{0}(t)}} \mathcal{A}_{n_{0}(l)}(t)-i \sum_{\substack{l^{\prime} \notin \Lambda_{s} l^{\prime} \neq l \\
\left|\omega_{l l^{\prime}}\right|<\Delta}}\left\langle\phi_{l}^{(0)}|\hat{W}| \phi_{l^{\prime}}^{(0)}\right\rangle e^{i \omega_{l l} t} \mathcal{A}_{l^{\prime}}(t) \\
& =\left\langle\phi_{l}^{(0)}|\hat{W}| \phi_{l}^{(0)}\right\rangle \mathcal{A}_{l}(t)+\left\langle\phi_{l}^{(0)}|\hat{W}| \phi_{n_{0}(l)}^{(0)}\right\rangle e^{i t \omega_{l n_{0}(l)}} \mathcal{A}_{n_{0}(l)}(t) \\
& -i \sum_{\substack{l^{\prime} \notin \Lambda_{s}, l^{\prime} \neq l \\
\left|\omega_{l l^{\prime}}\right|<\Delta}}\left\langle\phi_{l}^{(0)}|\hat{W}| \phi_{l^{\prime}}^{(0)}\right\rangle e^{i \omega_{l l^{\prime}} t} \int_{0}^{t} d \tau \sum_{\left|\omega_{l^{\prime} l^{\prime \prime}}\right|<\Delta}\left\langle\phi_{l^{\prime}}^{(0)}|\hat{W}| \phi_{l^{\prime \prime}}^{(0)}\right\rangle \mathcal{A}_{l^{\prime \prime}}(\tau) e^{i \omega_{l^{\prime} l^{\prime \prime}} \tau} \\
& =\left\langle\phi_{l}^{(0)}|\hat{W}| \phi_{l}^{(0)}\right\rangle \mathcal{A}_{l}(t)+\left\langle\phi_{l}^{(0)}|\hat{W}| \phi_{n_{0}(l)}^{(0)}\right\rangle e^{i t \omega_{l n_{0}(l)}} \mathcal{A}_{n_{0}(l)}(t) \\
& -i \int_{0}^{t} d \tau\left\{\sum_{\substack{l^{\prime} \notin \Lambda_{s}, l^{\prime} \neq l \\
\left|\omega_{l l^{\prime}}\right|<\Delta}} \sum_{\left|\omega_{l^{\prime} l^{\prime \prime}}\right|<\Delta}\left\langle\phi_{l}^{(0)}|\hat{W}| \phi_{l^{\prime}}^{(0)}\right\rangle\left\langle\phi_{l^{\prime}}^{(0)}|\hat{W}| \phi_{l^{\prime \prime}}^{(0)}\right\rangle e^{i \omega_{l l^{\prime}} t-i \omega_{l^{\prime \prime} l^{\prime}} \tau}\right\} \mathcal{A}_{l^{\prime \prime}}(\tau) .
\end{aligned}
$$

Now, the dominant terms inside the curly brackets will occur for $J=J^{\prime \prime}$ and $t \sim \tau$. By keeping these matrix elements and evaluating the amplitude at $t=\tau$, we obtain the equation

$i \frac{d}{d t} \mathcal{A}_{l \notin \Lambda_{s}}(t) \approx\left\langle\phi_{l}^{(0)}|\hat{W}| \phi_{n_{0}(l)}^{(0)}\right\rangle e^{i t \omega_{l_{n_{0}}(l)}} \mathcal{A}_{n_{0}(l)}(t)+z_{l}(t) \mathcal{A}_{l}(t)$,

where in this case $z_{l \notin \Lambda_{s}}(t)=\left\langle\phi_{l}^{(0)}|\hat{W}| \phi_{l}^{(0)}\right\rangle-i \int_{0}^{t} d \tau$

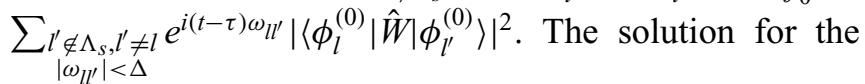
amplitude is

$$
\mathcal{A}_{l \notin \Lambda_{s}}(t) \approx-i\left\langle\phi_{l}^{(0)}|\hat{W}| \phi_{n_{0}(l)}^{(0)}\right\rangle \int_{0}^{t} e^{-i \mathcal{F}_{l}(t, \tau)} \mathcal{A}_{n_{0}(l)}(\tau) d \tau,
$$

where we define $\mathcal{F}_{l}(t, \tau)=-\tau \omega_{l, n_{0}(l)}+(t-\tau) \rho_{l l} \cdot \mathbf{h}-$ $i \mathbf{h}^{T} \cdot G_{l}(t, \tau) \cdot \mathbf{h}$. This is the expression for the amplitude we used in the main text. With this expression at hand, the disorder averaged quantity $\left[\mathcal{A}_{l^{\prime}}^{*}(t) \mathcal{A}_{l}(t)\right]$ we require is

$$
\begin{aligned}
{\left[\mathcal{A}_{l^{\prime}}^{*}(t) \mathcal{A}_{l}(t)\right] \approx } & \sum_{l l^{\prime}} \psi_{n_{0}\left(l^{\prime}\right)}^{*} \psi_{n_{0}(l)}\left(\rho_{l^{\prime} n_{0}\left(l^{\prime}\right)}^{*}\right)_{n^{\prime}}\left(\rho_{l, n_{0}(l)}\right)_{n} \\
& \times \int d^{2} \vec{\tau} e^{-i \tau_{2} \omega_{l^{\prime} n_{0}\left(l^{\prime}\right)}+i \tau_{1} \omega_{l n_{0}}(l)} \\
& \times\left[\mathbf{h}_{n^{\prime}} \mathbf{h}_{n} e^{-(1 / 2) \mathbf{h}^{T} \cdot \Gamma_{l^{\prime} l}(t, \vec{\tau}) \cdot \mathbf{h}} e^{i R_{l^{\prime} l^{\prime}}(t, \vec{\tau}) \cdot \mathbf{h}}\right],
\end{aligned}
$$

where $\Gamma_{l^{\prime} l}(t, \vec{\tau})=2\left[G_{l^{\prime}}^{\dagger}\left(t, \tau_{1}\right)+G_{l}\left(t, \tau_{2}\right)+G_{n_{0}\left(l^{\prime}\right)}^{\dagger}\left(\tau_{1}, 0\right)+\right.$ $\left.G_{n_{0}(l)}\left(\tau_{2}, 0\right)\right]$, and $R_{l^{\prime} l}(t, \vec{\tau})=\rho_{l^{\prime} l^{\prime}}\left(t-\tau_{2}\right)-\rho_{l l}\left(t-\tau_{1}\right)+$

$\rho_{n_{0}\left(l^{\prime}\right) n_{0}\left(l^{\prime}\right)} \tau_{2}-\rho_{n_{0}(l), n(l)} \tau_{1}$. Note that we can write

$$
\begin{aligned}
& {\left[\mathbf{h}_{n^{\prime}} \mathbf{h}_{n} e^{-(1 / 2) \mathbf{h}^{T} \cdot \Gamma_{l^{\prime} l}(t, \vec{\tau}) \cdot \mathbf{h}} e^{i R_{l^{\prime} l}(t, \vec{\tau}) \cdot \mathbf{h}}\right]} \\
& \quad=-\frac{\partial}{\partial\left(R_{l^{\prime} l}\right)_{n}} \frac{\partial}{\partial\left(R_{l^{\prime} l}\right)_{n^{\prime}}}\left[e^{-(1 / 2) \mathbf{h}^{T} \cdot \Gamma_{l^{\prime} l}(t, \vec{\tau}) \cdot \mathbf{h}} e^{i R_{l^{\prime} l}(t, \vec{\tau}) \cdot \mathbf{h}}\right],
\end{aligned}
$$

where the derivative is only with respect to diagonal matrix elements of the Pauli operators, so it does not act on the $\Gamma_{l^{\prime} l}(t, \vec{\tau})$ matrix. Thus, we need only disorder average the quantity

$$
\begin{aligned}
& {\left[e^{-(1 / 2) \mathbf{h}^{T} \cdot \Gamma_{l^{\prime} l}(t, \vec{\tau}) \cdot \mathbf{h}} e^{i R_{l^{\prime} l^{\prime}}(t, \vec{\tau}) \cdot \mathbf{h}}\right]} \\
& \quad=\frac{1}{\left(\pi W^{2} / 6\right)^{3 L / 2}} \int_{\mathbb{R}^{3 L}} d \mathbf{h} e^{-\left(6 / W^{2}\right) \mathbf{h}^{2}} e^{-(1 / 2) \mathbf{h}^{T} \cdot \Gamma_{l^{\prime} l}(t, \vec{\tau}) \cdot \mathbf{h}} \\
& \quad e^{i R_{l^{\prime} l}(t, \vec{\tau}) \cdot \mathbf{h}} \\
& \quad=\sqrt{\operatorname{det}\left\{\beta_{l^{\prime} l}(t, \vec{\tau})\right\}} e^{-\left(W^{2} / 24\right) R_{l^{\prime} l}^{T}\left(t, \tau, \tau^{\prime}\right) \cdot \beta_{l^{\prime} l}(t, \vec{\tau}) \cdot R_{l^{\prime} l^{\prime}}(t, \vec{\tau})} \\
& \quad=\mathcal{J}_{l^{\prime} l}(t, \vec{\tau}) .
\end{aligned}
$$

In these expressions, we define $\beta_{l^{\prime} l}(t, \vec{\tau})=\left[\mathbb{I}+\left(W^{2} / 12\right)\right.$ $\left.\Gamma_{l^{\prime} l}(t, \vec{\tau})\right]^{-1}$. The first and second derivative of this expression leads to

$$
\begin{aligned}
\frac{\partial}{\partial\left(R_{l^{\prime} l}\right)_{n}} \mathcal{J}_{l^{\prime l} l}(t, \vec{\tau})= & \mathcal{J}_{l^{\prime} l}(t, \vec{\tau})\left(-\frac{W^{2}}{24}\right) \Delta_{l^{\prime} l}^{n}(t, \vec{\tau}) \quad(\mathrm{C} 23) \\
= & \frac{\partial}{\partial\left(R_{l^{\prime}}\right)_{n^{\prime}}} \frac{\partial}{\partial\left(R_{l^{\prime}}\right)_{n}} \mathcal{J}_{l^{\prime} l}(t, \vec{\tau}) \mathcal{J}_{l^{\prime} l}(t, \vec{\tau}) \\
& \times\left(-\frac{W^{2}}{24}\right)\left(\left\{\beta_{l^{\prime} l}(t, \vec{\tau})\right\}_{l^{\prime}}^{l}+\left\{\beta_{l^{\prime} l}(t, \vec{\tau})\right\}_{l}^{l^{\prime}}\right)
\end{aligned}
$$




$$
+\mathcal{J}_{l^{\prime} l}(t, \vec{\tau})\left(-\frac{W^{2}}{24}\right)^{2} \Delta_{l^{\prime} l}^{n^{\prime}}(t, \vec{\tau}) \Delta_{l^{\prime} l}^{n}(t, \vec{\tau})
$$

with $\quad \Delta_{l^{\prime} l}^{n}(t, \vec{\tau})=\left\{\beta_{l^{\prime} l}(t, \vec{\tau}) \cdot R_{l^{\prime} l}(t, \vec{\tau})\right\}_{n}+\left\{R_{l^{\prime} l}^{T}(t, \vec{\tau})\right.$. $\left.\beta_{l^{\prime} l}(t, \vec{\tau})\right\}_{n}$. Putting it all together, the final expression is then

$$
\begin{aligned}
{\left[\mathcal{A}_{l^{\prime}}^{*}(t) \mathcal{A}_{l}(t)\right] \approx } & \psi_{n_{0}\left(l^{\prime}\right)}^{*} \psi_{n_{0}(l)} \frac{W^{2}}{24} \int d^{2} \vec{\tau} e^{-i \tau_{1} \omega_{l^{\prime} n_{0}\left(l^{\prime}\right)}+i \tau_{2} \omega_{l n_{0}}(l)} \sqrt{\operatorname{det}\left\{\beta_{l^{\prime} l}(t, \vec{\tau})\right\}} e^{-\left(W^{2} / 24\right) R_{l^{\prime} l}^{T}(t, \vec{\tau}) \cdot \beta_{l^{\prime} l^{\prime}}(t, \vec{\tau}) \cdot R_{l^{\prime} l^{\prime}}(t, \vec{\tau})} \\
& \times\left(\rho_{l^{\prime} n_{0}\left(l^{\prime}\right)}^{\dagger} \cdot \beta_{l^{\prime} l}(t, \vec{\tau}) \cdot \rho_{l n_{0}(l)}+\rho_{l n_{0}(l)}^{T} \cdot \beta_{l^{\prime} l}(t, \vec{\tau}) \cdot \rho_{l^{\prime} n_{0}\left(l^{\prime}\right)}^{*}-\frac{W^{2}}{24}\left\{\rho_{l^{\prime} n_{0}\left(l^{\prime}\right)}^{\dagger} \cdot \Delta_{l^{\prime} l}(t, \vec{\tau})\right\}\left\{\Delta_{l^{\prime} l}^{T}(t, \vec{\tau}) \cdot \rho_{l n_{0}(l)}\right\}\right),
\end{aligned}
$$

which is the result discussed in the main text.

\section{APPENDIX D: TEMPORAL CORRELATOR}

In this Appendix, we obtain the expression for temporal correlators discussed in the main text. We can capture the temporal correlations encoded in Eq. (25) by evaluating the spatial Fourier transform $\widetilde{\mathcal{C}_{a}}(t)=\sum_{R, r_{0}} \int d t e^{i \pi R} \mathcal{C}_{a}\left(r_{0}, r_{0}+\right.$ $R, t)=\left\langle\Psi\left|\left[\mathcal{P} \sigma_{\pi}^{a}(t) \mathcal{P}\right]\left[\mathcal{P} \sigma_{\pi}^{a}(0) \mathcal{P}\right]\right| \Psi\right\rangle-\left[\widetilde{\mathcal{M}}_{a}(\pi, t)\right] \widetilde{\mathcal{M}}_{a}$ $(\pi, 0)$. By expanding $|\Psi\rangle=\sum_{m} \psi_{J=1, m}\left|\phi_{J=1, m}^{(0)}\right\rangle$, where we use the notation $(\mathrm{Jm})$ that identifies towers in the spectrum, the action of $\mathcal{P} \sigma_{\pi}^{a}(0) \mathcal{P}$ on this state leads to

$$
\mathcal{P} \widetilde{\sigma}_{\pi}^{a}(0) \mathcal{P}\left|\mathbb{Z}_{2}\right\rangle \approx \sum_{m} \bar{\psi}_{J=1, m}^{a}\left|\phi_{J=1, m}^{(0)}\right\rangle \equiv\left|\Phi_{a}(0)\right\rangle,
$$

where $\bar{\psi}_{J=1, m}^{a}=\sum_{s, d} \psi_{1, m-s d} \Gamma_{1, m-s d}^{a, s d}$ with $s= \pm 1, d=$ 1,3 . For convenience, we define $\psi_{1, m}, \Gamma_{1, m}^{a, s d}=0$ whenever $m \notin\left[1, \mathcal{D}_{J}\right]$. Note that we have included matrix elements with $d=3$, which go beyond the tridiagonal in the matrix representation of $\sigma_{\pi}^{a}$. In contrast to the magnetization, for the calculation of temporal correlators we need matrix elements beyond the tridiagonal that are small but nevertheless contribute to the final temporal correlator.

Now, we have that $\left\langle\Psi\left|\left[\mathcal{P} \sigma_{\pi}^{a}(t) \mathcal{P}\right]\left[\mathcal{P} \sigma_{\pi}^{a}(0) \mathcal{P}\right]\right| \Psi\right\rangle=$ $\left\langle\Psi\left|\left[\mathcal{P} \sigma_{\pi}^{a}(t) \mathcal{P}\right]\right| \Phi_{a}(0)\right\rangle=\left\langle\Psi(t)\left|\sigma_{\pi}^{a}(0)\right| \Phi_{a}(t)\right\rangle$. The state $\left|\Phi_{a}(0)\right\rangle$ is then an un-normalized initial condition on which the unitary evolution operator acts. We can expand this time-evolved state in the form

$$
\left|\Phi_{a}(t)\right\rangle=\sum_{J m} \mathcal{B}_{J m}^{a}(t) e^{-i E_{J m}^{(0)} t}\left|\phi_{J m}^{(0)}\right\rangle
$$

This is formally the same starting point as for the calculation of the magnetization, implying that $\mathcal{B}_{J m}^{a}(t)=\left(\bar{\psi}_{n_{0}(J m)}^{a} / \psi_{n_{0}(J m)}\right) \mathcal{A}_{J m}(t)$. The correlator can thus be written in the form $\left[\widetilde{\mathcal{C}}_{a}(t)\right]=\left[\left\langle\Psi(t)\left|\widetilde{\sigma}_{\pi}^{a}\right| \Phi_{a}(t)\right\rangle\right]-$
$\left[\widetilde{\mathcal{M}}_{a}(\pi, t)\right] \widetilde{\mathcal{M}}_{a}(\pi, 0)$, where

$$
\begin{aligned}
{\left[\left\langle\Psi(t)\left|\widetilde{\sigma}_{\pi}^{a}\right| \Phi_{a}(t)\right\rangle\right] \approx } & \sum_{J m} \sum_{s d}\left(\frac{\bar{\psi}_{n_{0}(J m-s d)}^{a} \Gamma_{J m-s d}^{a, s d}}{\psi_{n_{0}(J m)}}\right) \\
& \times\left[\mathcal{A}_{J m+s d}^{*}(t) \mathcal{A}_{J m}(t)\right] e^{i t \omega_{J m+s d, J m}}
\end{aligned}
$$

This is the expression presented in Eq. (26) of the main text.

[1] P. Richerme, Z.-X. Gong, A. Lee, C. Senko, J. Smith, M. Foss-Feig, S. Michalakis, A. V. Gorshkov, and C. Monroe, Non-local propagation of correlations in quantum systems with long-range interactions, Nature 511, 198 (2014).

[2] P. Jurcevic, B. P. Lanyon, P. Hauke, C. Hempel, P. Zoller, R. Blatt, and C. F. Roos, Quasiparticle engineering and entanglement propagation in a quantum many-body system, Nature 511, 202 (2014).

[3] M. Gärttner, J. G. Bohnet, A. Safavi-Naini, M. L. Wall, J. J. Bollinger, and A. M. Rey, Measuring out-of-time-order correlations and multiple quantum spectra in a trapped-ion quantum magnet, Nat. Phys. 13, 781 (2017).

[4] H. Bernien, S. Schwartz, A. Keesling, H. Levine, A. Omran, H. Pichler, S. Choi, A. S. Zibrov, M. Endres, M. Greiner, V. Vuletic, and M. D. Lukin, Probing many-body dynamics on a 51-atom quantum simulator, Nature 551, 579 (2017).

[5] M. Srednicki, Chaos and quantum thermalization, Phys. Rev. E 50, 888 (1994).

[6] C. J. Turner, A. A. Michailidis, D. A. Abanin, M. Serbyn, and Z. Papic, Weak ergodicity breaking from quantum many-body scars, Nat. Phys. 14, 745 (2018).

[7] C. J. Turner, A. A. Michailidis, D. A. Abanin, M. Serbyn, and Z. Papić, Quantum scarred eigenstates in a rydberg atom chain: Entanglement, breakdown of thermalization, and stability to perturbations, Phys. Rev. B 98, 155134 (2018). 
[8] E. J. Heller, Bound-State Eigenfunctions of Classically Chaotic Hamiltonian Systems: Scars of Periodic Orbits, Phys. Rev. Lett. 53, 1515 (1984).

[9] G. G. de Polavieja, F. Borondo, and R. M. Benito, Scars in Groups of Eigenstates in a Classically Chaotic System, Phys. Rev. Lett. 73, 1613 (1994).

[10] S. Moudgalya, S. Rachel, B. A. Bernevig, and N. Regnault, Exact excited states of nonintegrable models, Phys. Rev. B 98, 235155 (2018).

[11] A. M. Alhambra, A. Anshu, and H. Wilming, Revivals imply quantum many-body scars, Phys. Rev. B 101, 205107 (2020).

[12] A. Hudomal, I. Vasić, N. Regnault, et al., Quantum scars of bosons with correlated hopping, Commun. Phys. 3, 99 (2020).

[13] S. Pai and M. Pretko, Dynamical Scar States in Driven Fracton Systems, Phys. Rev. Lett. 123, 136401 (2019).

[14] K. Bull, I. Martin, and Z. Papić, Systematic Construction of Scarred Many-Body Dynamics in 1D Lattice Models, Phys. Rev. Lett. 123, 030601 (2019).

[15] S. Ok, K. Choo, C. Mudry, C. Castelnovo, C. Chamon, and T. Neupert, Topological many-body scar states in dimensions one, two, and three, Phys. Rev. Res. 1, 033144 (2019).

[16] B. Mukherjee, S. Nandy, A. Sen, D. Sen, and K. Sengupta, Collapse and revival of quantum many-body scars via floquet engineering, Phys. Rev. B 101, 245107 (2020).

[17] D. K. Mark, C.-J. Lin, and O. I. Motrunich, Unified structure for exact towers of scar states in the Affleck-KennedyLieb-Tasaki and other models, Phys. Rev. B 101, 195131 (2020).

[18] H. Zhao, J. Vovrosh, F. Mintert, and J. Knolle, Quantum Many-Body Scars in Optical Lattices, Phys. Rev. Lett. 124, 160604 (2020).

[19] A. A. Michailidis, C. J. Turner, Z. Papić, D. A. Abanin, and M. Serbyn, Stabilizing two-dimensional quantum scars by deformation and synchronization, Phys. Rev. Res. 2, 022065(R) (2020).

[20] B. van Voorden, J. c. v. Minář, and K. Schoutens, Quantum many-body scars in transverse field Ising ladders and beyond, Phys. Rev. B 101, 220305(R) (2020).

[21] K. Lee, R. Melendrez, A. Pal, and H. J. Changlani, Exact three-colored quantum scars from geometric frustration, Phys. Rev. B 101, 241111(R) (2020).

[22] N. Shiraishi and T. Mori, Systematic Construction of Counterexamples to the Eigenstate Thermalization Hypothesis, Phys. Rev. Lett. 119, 030601 (2017).

[23] S. Moudgalya, N. Regnault, and B. A. Bernevig, Entanglement of exact excited states of Affleck-Kennedy-LiebTasaki models: Exact results, many-body scars, and violation of the strong eigenstate thermalization hypothesis, Phys. Rev. B 98, 235156 (2018).

[24] C.-J. Lin and O. I. Motrunich, Exact Quantum Many-Body Scar States in the Rydberg-Blockaded Atom Chain, Phys. Rev. Lett. 122, 173401 (2019).

[25] S. Chattopadhyay, H. Pichler, M. D. Lukin, and W. W. Ho, Quantum many-body scars from virtual entangled pairs, Phys. Rev. B 101, 174308 (2020).
[26] K. Mizuta, K. Takasan, and N. Kawakami, Exact Floquet quantum many-body scars under Rydberg blockade, Phys. Rev. Res. 2, 033284 (2020).

[27] S. Moudgalya, N. Regnault, and B. A. Bernevig, $\eta$-pairing in Hubbard models: From spectrum generating algebras to quantum many-body scars, Phys. Rev. B 102, 085140 (2020).

[28] S. Moudgalya, E. O’Brien, B. A. Bernevig, P. Fendley, and N. Regnault, Large classes of quantum scarred Hamiltonians from matrix product states, Phys. Rev. B 102, 085120 (2020).

[29] T. Iadecola and M. Schecter, Quantum many-body scar states with emergent kinetic constraints and finiteentanglement revivals, Phys. Rev. B 101, 024306 (2020).

[30] S. Choi, C. J. Turner, H. Pichler, W. W. Ho, A. A. Michailidis, Z. Papić, M. Serbyn, M. D. Lukin, and D. A. Abanin, Emergent SU(2) Dynamics and Perfect Quantum ManyBody Scars, Phys. Rev. Lett. 122, 220603 (2019).

[31] K. Bull, J.-Y. Desaules, and Z. Papić, Quantum scars as embeddings of weakly broken Lie algebra representations, Phys. Rev. B 101, 165139 (2020).

[32] N. O'Dea, F. Burnell, A. Chandran, and V. Khemani, arXiv preprint arXiv:2007.16207 (2020).

[33] B. Buča, J. Tindall, and D. Jaksch, Double-slit photoelectron interference in strong-field ionization of the neon dimer, Nat. Commun. 10, 1 (2019).

[34] M. Medenjak, B. Buča, and D. Jaksch, Isolated Heisenberg magnet as a quantum time crystal, Phys. Rev. B 102, 041117(R) (2020).

[35] W. W. Ho, S. Choi, H. Pichler, and M. D. Lukin, Periodic Orbits, Entanglement, and Quantum Many-Body Scars in Constrained Models: Matrix Product State Approach, Phys. Rev. Lett. 122, 040603 (2019).

[36] V. Khemani, C. R. Laumann, and A. Chandran, Signatures of integrability in the dynamics of Rydberg-blockaded chains, Phys. Rev. B 99, 161101(R) (2019).

[37] C. J. Turner, J.-Y. Desaules, K. Bull, and Z. Papić, 'Correspondence principle for many-body scars in ultracold Rydberg atoms,' (2020), arXiv:2006.13207.

[38] A. A. Michailidis, C. J. Turner, Z. Papić, D. A. Abanin, and M. Serbyn, Slow Quantum Thermalization and ManyBody Revivals from Mixed Phase Space, Phys. Rev. X 10, 011055 (2020).

[39] J. J. Burnett, A. Bengtsson, M. Scigliuzzo, D. Niepce, M. Kudra, P. Delsing, and J. Bylander, Decoherence benchmarking of superconducting qubits, npj Quantum Inf. 5, 54 (2019).

[40] S. S. Tannu and M. K. Qureshi, Proceedings of the TwentyFourth International Conference on Architectural Support for Programming Languages and Operating Systems (2019).

[41] J. Lisenfeld, A. Bilmes, A. Megrant, R. Barends, J. Kelly, P. Klimov, G. Weiss, J. M. Martinis, and A. V. Ustinov, Electric field spectroscopy of material defects in transmon qubits, npj Quantum Inf. 5, 105 (2019).

[42] C. Berke, E. Varvelis, S. Trebst, A. Altland, and D. P. DiVincenzo, arXiv preprint arXiv:2012.05923 (2020).

[43] J. Smith, A. Lee, P. Richerme, B. Neyenhuis, P. W. Hess, P. Hauke, M. Heyl, D. A. Huse, and C. Monroe, Many-body 
localization in a quantum simulator with programmable random disorder, Nat. Phys. 12, 907 (2016).

[44] J. Zhang, P. W. Hess, A. Kyprianidis, P. Becker, A. Lee, J. Smith, G. Pagano, I.-D. Potirniche, A. C. Potter, A. Vishwanath, N. Y. Yao, and C. Monroe, Observation of a discrete time crystal, Nature 543, 217 (2017).

[45] M. Marcuzzi, J. c. v. Minář, D. Barredo, S. de Léséleuc, H. Labuhn, T. Lahaye, A. Browaeys, E. Levi, and I. Lesanovsky, Facilitation Dynamics and Localization Phenomena in Rydberg Lattice Gases with Position Disorder, Phys. Rev. Lett. 118, 063606 (2017).

[46] D. Basko, I. Aleiner, and B. Altshuler, Metal-insulator transition in a weakly interacting many-electron system with localized single-particle states, Ann. Phys. 321, 1126 (2006).

[47] V. Oganesyan and D. A. Huse, Localization of interacting fermions at high temperature, Phys. Rev. B 75, 155111 (2007).

[48] A. Pal and D. A. Huse, Many-body localization phase transition, Phys. Rev. B 82, 174411 (2010).

[49] M. Serbyn, Z. Papić, and D. A. Abanin, Local Conservation Laws and the Structure of the Many-Body Localized States, Phys. Rev. Lett. 111, 127201 (2013).

[50] M. Schreiber, S. S. Hodgman, P. Bordia, H. P. Lüschen, M. H. Fischer, R. Vosk, E. Altman, U. Schneider, and I. Bloch, Observation of many-body localization of interacting fermions in a quasirandom optical lattice, Science 349 , 842 (2015).

[51] R. Nandkishore and D. A. Huse, Many-body localization and thermalization in quantum statistical Mechanics, Annu. Rev. Condens. Matter Phys. 6, 15 (2015).

[52] J.-y. Choi, S. Hild, J. Zeiher, P. Schauß, A. Rubio-Abadal, T. Yefsah, V. Khemani, D. A. Huse, I. Bloch, and C. Gross, Exploring the many-body localization transition in two dimensions, Science 352, 1547 (2016).

[53] C. W. von Keyserlingk, V. Khemani, and S. L. Sondhi, Absolute stability and spatiotemporal long-range order in Floquet systems, Phys. Rev. B 94, 085112 (2016).

[54] N. Y. Yao, A. C. Potter, I.-D. Potirniche, and A. Vishwanath, Discrete Time Crystals: Rigidity, Criticality, and Realizations, Phys. Rev. Lett. 118, 030401 (2017).

[55] S. Choi, J. Choi, R. Landig, G. Kucsko, H. Zhou, J. Isoya, F. Jelezko, S. Onoda, H. Sumiya, V. Khemani, C. von Keyserlingk, N. Y. Yao, E. Demler, and M. D. Lukin, Observation of discrete time-crystalline order in a disordered dipolar many-body system, Nature 543, 221 (2017).

[56] M. Schecter and T. Iadecola, Weak Ergodicity Breaking and Quantum Many-Body Scars in Spin-1 XY Magnets, Phys. Rev. Lett. 123, 147201 (2019).

[57] N. Shibata, N. Yoshioka, and H. Katsura, Onsager's Scars in Disordered Spin Chains, Phys. Rev. Lett. 124, 180604 (2020).

[58] N. S. Srivatsa, J. Wildeboer, A. Seidel, and A. E. B. Nielsen, 'Quantum many-body scars with chiral topological order in 2d and critical properties in 1d,' (2020), arXiv:2009.03923.

[59] C. Chen, F. Burnell, and A. Chandran, How Does a Locally Constrained Quantum System Localize? Phys. Rev. Lett. 121, 085701 (2018).

[60] M. V. Berry, Regular and irregular semiclassical wavefunctions, J. Phys. A: Math. Gen. 10, 2083 (1977).
[61] S. Sridhar, Experimental Observation of Scarred Eigenfunctions of Chaotic Microwave Cavities, Phys. Rev. Lett. 67, 785 (1991).

[62] E. J. Heller, The Semiclassical Way to Dynamics and Spectroscopy (Princeton University Press, Princeton, NJ, 2018).

[63] M. Saffman, T. G. Walker, and K. Mølmer, Quantum information with Rydberg atoms, Rev. Mod. Phys. 82, 2313 (2010).

[64] R. Löw, H. Weimer, J. Nipper, J. B. Balewski, B. Butscher, H. P. Büchler, and T. Pfau, An experimental and theoretical guide to strongly interacting Rydberg gases, J. Phys. B: At., Mol. Opt. Phys. 45, 113001 (2012).

[65] T. Iadecola, M. Schecter, and S. Xu, Quantum manybody scars from magnon condensation, Phys. Rev. B 100, 184312 (2019).

[66] Y. Y. Atas, E. Bogomolny, O. Giraud, and G. Roux, Distribution of the Ratio of Consecutive Level Spacings in Random Matrix Ensembles, Phys. Rev. Lett. 110, 084101 (2013).

[67] I. Mondragon-Shem, M. Vavilov, and I. Martin, (In preparation).

[68] J. Rovny, R. L. Blum, and S. E. Barrett, Observation of Discrete-Time-Crystal Signatures in an Ordered Dipolar Many-Body System, Phys. Rev. Lett. 120, 180603 (2018).

[69] C. Cohen-Tannoudji, B. Diu, and F. Laloë, Quantum Mechanics (Wiley, New York, NY, 1991).

[70] C.-J. Lin, A. Chandran, and O. I. Motrunich, Slow thermalization of exact quantum many-body scar states under perturbations, Phys. Rev. Res. 2, 033044 (2020).

[71] V. Khemani, A. Lazarides, R. Moessner, and S. L. Sondhi, Phase Structure of Driven Quantum Systems, Phys. Rev. Lett. 116, 250401 (2016).

[72] D. V. Else, B. Bauer, and C. Nayak, Floquet Time Crystals, Phys. Rev. Lett. 117, 090402 (2016).

[73] O. Viehmann, J. von Delft, and F. Marquardt, Observing the Nonequilibrium Dynamics of the Quantum TransverseField Ising Chain in Circuit QED, Phys. Rev. Lett. 110, 030601 (2013).

[74] C. Monroe, W. C. Campbell, L. M. Duan, Z. X. Gong, A. V. Gorshkov, P. Hess, R. Islam, K. Kim, N. Linke, G. Pagano, P. Richerme, C. Senko, and N. Y. Yao, 'Programmable quantum simulations of spin systems with trapped ions,' (2019), arXiv:1912.07845.

[75] J. I. Cirac and P. Zoller, Quantum Computations with Cold Trapped Ions, Phys. Rev. Lett. 74, 4091 (1995).

[76] C. D. Bruzewicz, J. Chiaverini, R. McConnell, and J. M. Sage, Trapped-ion quantum computing: Progress and challenges, Appl. Phys. Rev. 6, 021314 (2019).

[77] A. Smith, M. S. Kim, F. Pollmann, and J. Knolle, Simulating quantum many-body dynamics on a current digital quantum computer, npj Quantum Inf. 5, 106 (2019).

[78] K. Wright et al., Benchmarking an 11-qubit quantum computer, Nat. Commun. 10, 5464 (2019).

[79] Y. Nam et al., Ground-state energy estimation of the water molecule on a trapped-ion quantum computer, npj Quantum Inf. 6, 33 (2020).

[80] M. Kjaergaard, M. E. Schwartz, J. Braumüller, P. Krantz, J. I.-J. Wang, S. Gustavsson, and W. D. Oliver, 
Superconducting qubits: Current state of Play, Annu. Rev. Cond. Matter Phys. 11, 369 (2020).

[81] C. Cîrstoiu, Z. Holmes, J. Iosue, L. Cincio, P. J. Coles, and A. Sornborger, Variational fast forwarding for quantum simulation beyond the coherence time, npj Quantum Inf. 6, $82(2020)$.

[82] V. Giovannetti, S. Lloyd, and L. Maccone, Quantum Metrology, Phys. Rev. Lett. 96, 010401 (2006).
[83] L. Pezzè, A. Smerzi, M. K. Oberthaler, R. Schmied, and P. Treutlein, Quantum metrology with nonclassical states of atomic ensembles, Rev. Mod. Phys. 90, 035005 (2018).

[84] S. Dooley, arXiv preprint arXiv:2101.04670 (2021).

[85] C. Rigetti and M. Devoret, Fully microwave-tunable universal gates in superconducting qubits with linear couplings and fixed transition frequencies, Phys. Rev. B 81, 134507 (2010). 